ANL-HEP-PR-05-109

CERN-PH-TH/2005-158

DCPT $/ 05 / 128$

EFI-05-19

FERMILAB-Pub-05-370-T

$\operatorname{IPPP} / 05 / 64$

hep-ph/0511023

\title{
MSSM Higgs Boson Searches at the Tevatron and the LHC: Impact of Different Benchmark Scenarios
}

\author{
M. Carena ${ }^{1 *}$, S. Heinemeyer ${ }^{2 \dagger}$, C.E.M. WagneR ${ }^{3,4 \ddagger}$ And G. Weiglein ${ }^{5 \S}$ \\ ${ }^{1}$ Theoretical Physics Dept., Fermilab, Batavia, IL 60510-0500, USA \\ ${ }^{2}$ CERN TH Division, Dept. of Physics, CH-1211 Geneva 23, Switzerland \\ ${ }^{3}$ HEP Division, Argonne Natl. Lab., 9700 Cass Ave., Argonne, IL 60439, USA \\ ${ }^{4}$ Enrico Fermi Institute, Univ. of Chicago, 5640 Ellis Ave., Chicago, IL 60637, USA \\ ${ }^{5}$ IPPP, University of Durham, Durham DH1 3LE, UK
}

\begin{abstract}
The Higgs boson search has shifted from LEP2 to the Tevatron and will subsequently move to the LHC. The current limits from the Tevatron and the prospective sensitivities at the LHC are often interpreted in specific MSSM scenarios. For heavy Higgs boson production and subsequent decay into $b \bar{b}$ or $\tau^{+} \tau^{-}$, the present Tevatron data allow to set limits in the $M_{A}$-tan $\beta$ plane for small $M_{A}$ and large $\tan \beta$ values. Similar channels have been explored for the LHC, where the discovery reach extends to higher values of $M_{A}$ and smaller $\tan \beta$. Searches for MSSM charged Higgs bosons, produced in top decays or in association with top quarks, have also been investigated at the Tevatron and the LHC. We analyze the current Tevatron limits and prospective LHC sensitivities. We discuss how robust they are with respect to variations of the other MSSM parameters and possible improvements of the theoretical predictions for Higgs boson production and decay. It is shown that the inclusion of supersymmetric radiative corrections to the production cross sections and decay widths leads to important modifications of the present limits on the MSSM parameter space. The impact on the region where only the lightest MSSM Higgs boson can be detected at the LHC is also analyzed. We propose to extend the existing benchmark scenarios by including additional values of the higgsino mass parameter $\mu$. This affects only slightly the search channels for a SM-like Higgs boson, while having a major impact on the searches for non-standard MSSM Higgs bosons.
\end{abstract}

*email: carena@fnal.gov

$\dagger$ †email: Sven.Heinemeyer@cern.ch

${ }_{\ddagger}^{\ddagger}$ email: cwagner@hep.anl.gov

§email: Georg.Weiglein@durham.ac.uk 


\section{Introduction}

Disentangling the mechanism that controls electroweak symmetry breaking is one of the main tasks of the current and next generation of colliders. Among the most studied candidates in the literature are the Higgs mechanism within the Standard Model (SM) or within the Minimal Supersymmetric Standard Model (MSSM). Contrary to the SM, two Higgs doublets are required in the MSSM, resulting in five physical Higgs boson degrees of freedom. In the absence of explicit $\mathcal{C P}$-violation in the soft supersymmetry-breaking terms these are the light and heavy $\mathcal{C P}$-even Higgs bosons, $h$ and $H$, the $\mathcal{C P}$-odd Higgs boson, $A$, and the charged Higgs boson, $H^{ \pm}$. The Higgs sector of the MSSM can be specified at lowest order in terms of $M_{Z}, M_{A}$, and $\tan \beta \equiv v_{2} / v_{1}$, the ratio of the two Higgs vacuum expectation values. The masses of the $\mathcal{C P}$-even neutral Higgs bosons and the charged Higgs boson can be calculated, including higher-order corrections, in terms of the other MSSM parameters.

After the termination of LEP in the year 2000 (the close-to-final LEP results can be found in Refs. [1,2]), the Higgs boson search has shifted to the Tevatron and will later be continued at the LHC. Due to the large number of free parameters, a complete scan of the MSSM parameter space is too involved. Therefore the search results at LEP have been interpreted [2] in several benchmark scenarios [3,4]. Current analyses at the Tevatron and investigations of the LHC potential also have been performed in the scenarios proposed in Refs. [3,4]. The $m_{h}^{\max }$ scenario has been used to obtain conservative bounds on $\tan \beta$ for fixed values of the top-quark mass and the scale of the supersymmetric particles [5]. Besides the $m_{h}^{\max }$ scenario and the no-mixing scenario, where a vanishing mixing in the stop sector is assumed, the "small $\alpha_{\text {eff }}$ " scenario and the "gluophobic Higgs scenario" have been investigated [6]. While the latter one exhibits a strong suppression of the $g g h$ coupling over large parts of the $M_{A^{-}}$ $\tan \beta$ parameter space, the small $\alpha_{\text {eff }}$ scenario has strongly reduced couplings of the light $\mathcal{C P}$-even Higgs boson to bottom-type fermions up to $M_{A} \lesssim 350 \mathrm{GeV}$. These scenarios are conceived to study particular cases of challenging and interesting phenomenology in the searches for the SM-like Higgs boson, i.e. mostly the light $\mathcal{C} \mathcal{P}$-even Higgs boson.

The current searches at the Tevatron are not yet sensitive to a SM-like Higgs in the mass region allowed by the LEP exclusion bounds $[1,2]$. On the other hand, scenarios with enhanced Higgs boson production cross sections can be probed already with the currently accumulated luminosity. Enhanced production cross sections can occur in particular for low $M_{A}$ in combination with large $\tan \beta$ due to the enhanced couplings of the Higgs bosons to down-type fermions. The corresponding limits on the Higgs production cross section times branching ratio of the Higgs decay into down-type fermions can be interpreted in MSSM benchmark scenarios. Limits from Run II of the Tevatron have recently been published for the following channels [7-9] (here and in the following $\phi$ denotes all three neutral MSSM Higgs bosons, $\phi=h, H, A)$ :

$$
\begin{aligned}
& b \bar{b} \phi, \phi \rightarrow b \bar{b} \text { (with one additional tagged } b \text { jet), } \\
& p \bar{p} \rightarrow \phi \rightarrow \tau^{+} \tau^{-} \text {(inclusive), } \\
& p \bar{p} \rightarrow t \bar{t} \rightarrow H^{ \pm} W^{\mp} b \bar{b}, H^{ \pm} \rightarrow \tau \nu_{\tau} .
\end{aligned}
$$

The obtained cross section limits have been interpreted in the $m_{h}^{\max }$ and the no-mixing scenario with a value for the higgsino mass parameter of $\mu=-200 \mathrm{GeV}$ [7] and $\mu=$ 
$\pm 200 \mathrm{GeV}$ [8]. In these scenarios for $M_{A} \approx 100 \mathrm{GeV}$ the limits on $\tan \beta$ are $\tan \beta \lesssim 50$.

In this article, we investigate the dependence of the CDF and D0 exclusion bounds in the $M_{A}-\tan \beta$ plane on the parameters entering through the most relevant supersymmetric radiative corrections in the theoretical predictions for Higgs boson production and decay processes. We will show that the bounds obtained from the $b \bar{b} \phi, \phi \rightarrow b \bar{b}$ channel depend very sensitively on the radiative corrections affecting the relation between the bottom quark mass and the bottom Yukawa coupling. ${ }^{1}$ In the channels with $\tau^{+} \tau^{-}$final states, on the other hand, compensations between large corrections in the Higgs production and the Higgs decay occur. In this context we investigate the impact of a large radiative correction in the $g g \rightarrow \phi$ production process that had previously been omitted.

In order to reflect the impact of the corrections to the bottom Yukawa coupling on the exclusion bounds we suggest to supplement the existing $m_{h}^{\max }$ and no-mixing scenarios, mostly designed to search for the light $\mathcal{C P}$-even MSSM Higgs boson, $h$, with additional values for the higgsino mass parameter $\mu$. In fact, varying the value and sign of $\mu$, while keeping fixed the values of the gluino mass and the common third generation squark mass parameter $M_{\mathrm{SUSY}}$, demonstrates the effect of the radiative corrections on the production and decay processes. The scenarios discussed here are designed specifically to study the MSSM Higgs sector without assuming any particular soft supersymmetry-breaking scenario and taking into account constraints only from the Higgs boson sector itself. In particular, constraints from requiring the correct cold dark matter density, $\operatorname{BR}(b \rightarrow s \gamma)$ or $(g-2)_{\mu}$, which depend on other parameters of the theory, are not crucial in defining the Higgs boson sector, and may be avoided. However, we also include a brief discussion of the "constrained$m_{h}^{\max }$ " scenario, which in the case of minimal flavor violation and positive values of $\mu$ leads to a better agreement with the constraints from $\mathrm{BR}(b \rightarrow s \gamma)$.

We also study the non-standard MSSM Higgs boson search sensitivity at the LHC, focusing on the processes $p p \rightarrow H / A+X, H / A \rightarrow \tau^{+} \tau^{-}$and $p p \rightarrow t H^{ \pm}+X, H^{ \pm} \rightarrow \tau \nu_{\tau}$, and stress the relevance of the proper inclusion of supersymmetric radiative corrections to the production cross sections and decay widths. We show the impact of these corrections by investigating the variation of the Higgs boson discovery reach in the benchmark scenarios for different values of $\mu$. In particular, we discuss the resulting modification of the parameter region in which only the light $\mathcal{C} \mathcal{P}$-even MSSM Higgs boson can be detected at the LHC.

The paper is organized as follows: Section 2 gives a summary of the most relevant supersymmetric radiative corrections to the Higgs boson production cross section and decay widths, while also introducing our notation. In section 3 we analyze the impact of these radiative corrections on the current Tevatron limits in the large $\tan \beta$ region, as well as on the future LHC reach for the heavy, non-standard, MSSM Higgs bosons. In section 4, based on the results of section 3, we propose an extension of the existing benchmark scenarios. The conclusions are presented in section 5 .

\footnotetext{
${ }^{1}$ We concentrate here on the effects of supersymmetric radiative corrections. For a recent account of uncertainties related to parton distribution functions, see e.g. Ref. [10].
} 


\section{Predictions for Higgs boson production and decay processes}

\subsection{Notation and renormalization}

The tree-level values for the $\mathcal{C P}$-even Higgs bosons of the MSSM, $m_{h}$ and $m_{H}$, are determined by $\tan \beta$, the $\mathcal{C} \mathcal{P}$-odd Higgs-boson mass $M_{A}$, and the $Z$ boson mass $M_{Z}$. The mass of the charged Higgs boson, $M_{H^{ \pm}}$, is given in terms of $M_{A}$ and the $W$ boson mass, $M_{W}$. Beyond the tree-level, the main correction to the Higgs boson masses stems from the $t / \tilde{t}$ sector, and for large values of $\tan \beta$ also from the $b / \tilde{b}$ sector.

In order to fix our notations, we list the conventions for the inputs from the scalar top and scalar bottom sector of the MSSM: the mass matrices in the basis of the current eigenstates $\tilde{t}_{L}, \tilde{t}_{R}$ and $\tilde{b}_{L}, \tilde{b}_{R}$ are given by

$$
\begin{aligned}
\mathcal{M}_{\tilde{t}}^{2} & =\left(\begin{array}{cc}
M_{\tilde{t}_{L}}^{2}+m_{t}^{2}+\cos 2 \beta\left(\frac{1}{2}-\frac{2}{3} s_{\mathrm{w}}^{2}\right) M_{Z}^{2} & m_{t} X_{t} \\
m_{t} X_{t} & M_{\tilde{t}_{R}}^{2}+m_{t}^{2}+\frac{2}{3} \cos 2 \beta s_{\mathrm{w}}^{2} M_{Z}^{2}
\end{array}\right), \\
\mathcal{M}_{\tilde{b}}^{2} & =\left(\begin{array}{cc}
M_{\tilde{b}_{L}}^{2}+m_{b}^{2}+\cos 2 \beta\left(-\frac{1}{2}+\frac{1}{3} s_{\mathrm{w}}^{2}\right) M_{Z}^{2} & m_{b} X_{b} \\
m_{b} X_{b} & M_{\tilde{b}_{R}}^{2}+m_{b}^{2}-\frac{1}{3} \cos 2 \beta s_{\mathrm{w}}^{2} M_{Z}^{2}
\end{array}\right),
\end{aligned}
$$

where

$$
m_{t} X_{t}=m_{t}\left(A_{t}-\mu \cot \beta\right), \quad m_{b} X_{b}=m_{b}\left(A_{b}-\mu \tan \beta\right) .
$$

Here $A_{t}$ denotes the trilinear Higgs-stop coupling, $A_{b}$ denotes the Higgs-sbottom coupling, and $\mu$ is the higgsino mass parameter.

$\mathrm{SU}(2)$ gauge invariance leads to the relation

$$
M_{\tilde{t}_{L}}=M_{\tilde{b}_{L}} .
$$

For the numerical evaluation, a convenient choice is

$$
M_{\tilde{t}_{L}}=M_{\tilde{b}_{L}}=M_{\tilde{t}_{R}}=M_{\tilde{b}_{R}}=: M_{\mathrm{SUSY}} .
$$

Concerning analyses for the case where $M_{\tilde{t}_{R}} \neq M_{\tilde{t}_{L}} \neq M_{\tilde{b}_{R}}$, see e.g. Refs. [11,12]. It has been shown that the upper bound on $m_{h}$ obtained using eq. (8) is the same as for the more general case, provided that $M_{\mathrm{SUSY}}$ is identified with the heaviest mass of $M_{\tilde{t}_{R}}, M_{\tilde{t}_{L}}, M_{\tilde{b}_{R}}[12]$. We furthermore use the short-hand notation

$$
M_{S}^{2}:=M_{\mathrm{SUSY}}^{2}+m_{t}^{2} .
$$

Accordingly, the most important parameters for the corrections in the Higgs sector are $m_{t}, M_{\mathrm{SuSY}}, X_{t}$, and $X_{b}$. The Higgs sector observables furthermore depend on the $\mathrm{SU}(2)$ gaugino mass parameter, $M_{2}$. The other gaugino mass parameter, $M_{1}$, is usually fixed via the GUT relation

$$
M_{1}=\frac{5}{3} \frac{s_{\mathrm{w}}^{2}}{c_{\mathrm{w}}^{2}} M_{2} .
$$

At the two-loop level also the gluino mass, $m_{\tilde{g}}$, enters the predictions for the Higgs-boson masses. 
Corrections to the MSSM Higgs boson sector have been evaluated in several approaches. The status of the available corrections to the masses and mixing angles in the MSSM Higgs sector (with real parameters) can be summarized as follows. For the one-loop part, the complete result within the MSSM is known [13-16]. The by far dominant one-loop contribution is the $\mathcal{O}\left(\alpha_{t}\right)$ term due to top and stop loops $\left(\alpha_{t} \equiv h_{t}^{2} /(4 \pi), h_{t}\right.$ being the top-quark Yukawa coupling). Concerning the two-loop effects, their computation is quite advanced and has now reached a stage such that all the presumably dominant contributions are known [12,17-31]. They include (evaluated for vanishing external momenta) the strong corrections, usually indicated as $\mathcal{O}\left(\alpha_{t} \alpha_{s}\right)$, and Yukawa corrections, $\mathcal{O}\left(\alpha_{t}^{2}\right)$, to the dominant one-loop $\mathcal{O}\left(\alpha_{t}\right)$ term, as well as the strong corrections to the bottom/sbottom one-loop $\mathcal{O}\left(\alpha_{b}\right)$ term $\left(\alpha_{b} \equiv h_{b}^{2} /(4 \pi)\right)$, i.e. the $\mathcal{O}\left(\alpha_{b} \alpha_{s}\right)$ contribution. The latter can be relevant for large values of $\tan \beta$. For the (s)bottom corrections the all-order resummation of the $\tan \beta$-enhanced terms, $\mathcal{O}\left(\alpha_{b}\left(\alpha_{s} \tan \beta\right)^{n}\right)$, has also been computed. Recently the $\mathcal{O}\left(\alpha_{t} \alpha_{b}\right)$ and $\mathcal{O}\left(\alpha_{b}^{2}\right)$ corrections have been obtained. The remaining theoretical uncertainty on the light $\mathcal{C P}$-even Higgs boson mass has been estimated to be below $\sim 3 \mathrm{GeV}[32,33]$. The above calculations have been implemented into public codes. The program FeynHiggs [34-37] is based on the results obtained in the Feynman-diagrammatic (FD) approach $[12,21,31,32]$. It includes all the above corrections. The code CPsuperH [38] is based on the renormalization group (RG) improved effective potential approach $[18,19,39]$. For the MSSM with real parameters the two codes can differ by up to $\sim 4 \mathrm{GeV}$ for the light $\mathcal{C} \mathcal{P}$-even Higgs boson mass, mostly due to formally subleading two-loop corrections that are included only in FeynHiggs. For the MSSM with complex parameters the phase dependence at the two-loop level is included in a more advanced way [40] in CPsuperH, but, on the other hand, CPsuperH does not contain all the subleading one-loop contributions that are included [41,42] in FeynHiggs. Most recently a full two-loop effective potential calculation (including even the momentum dependence for the leading pieces) has been published [43]. However, no computer code is publicly available. In the following we will concentrate on the MSSM with real parameters.

It should be noted in this context that the FD result has been obtained in the on-shell (OS) renormalization scheme, whereas the $\mathrm{RG}$ result has been calculated using the $\overline{\mathrm{MS}}$ scheme; see Refs. $[39,44]$ for a detailed comparison. Owing to the different schemes used in the FD and the RG approach for the renormalization in the scalar top sector, the parameters $X_{t}$ and $M_{\mathrm{SUSY}}$ are also scheme-dependent in the two approaches. This difference between the corresponding parameters has to be taken into account when comparing the results of the two approaches. In a simple approximation the relation between the parameters in the different schemes is at $\mathcal{O}\left(\alpha_{s}\right)$ given by [39]

$$
\begin{aligned}
M_{S}^{2, \overline{\mathrm{MS}}} & \approx M_{S}^{2, \mathrm{OS}}-\frac{8}{3} \frac{\alpha_{s}}{\pi} M_{S}^{2}, \\
X_{t}^{\overline{\mathrm{MS}}} & \approx X_{t}^{\mathrm{OS}}+\frac{\alpha_{s}}{3 \pi} M_{S}\left(8+4 \frac{X_{t}}{M_{S}}-3 \frac{X_{t}}{M_{S}} \log \left(\frac{m_{t}^{2}}{M_{S}^{2}}\right)\right),
\end{aligned}
$$

where in the terms proportional to $\alpha_{s}$ it is not necessary to distinguish between $\overline{\mathrm{MS}}$ and onshell quantities, since the difference is of higher order. The $\overline{\mathrm{MS}}$ top-quark mass, $m_{t}^{\overline{\mathrm{MS}}}\left(m_{t}\right) \equiv$ $\bar{m}_{t}$, is related to the top-quark pole mass, $m_{t}^{\mathrm{OS}} \equiv m_{t}$, in $\mathcal{O}\left(\alpha_{s}\right)$ by

$$
\bar{m}_{t}=\frac{m_{t}}{1+\frac{4}{3 \pi} \alpha_{s}\left(m_{t}\right)} \text {. }
$$


While the resulting shift in the parameter $M_{\text {SUSY }}$ turns out to be relatively small in general, sizable differences can occur between the numerical values of $X_{t}$ in the two schemes, see Refs. $[12,39]$. For this reason we specify below different values for $X_{t}$ within the two approaches.

\subsection{Leading effects from the bottom/sbottom sector}

The relation between the bottom-quark mass and the Yukawa coupling $h_{b}$, which controls also the interaction between the Higgs fields and the sbottom quarks, reads at lowest order $m_{b}=h_{b} v_{1}$. This relation is affected at one-loop order by large radiative corrections $[28-30,45]$, proportional to $h_{b} v_{2}$, in general giving rise to $\tan \beta$-enhanced contributions. These terms proportional to $v_{2}$, often called threshold corrections to the bottom mass, are generated either by gluino-sbottom one-loop diagrams (resulting in $\mathcal{O}\left(\alpha_{b} \alpha_{s}\right)$ corrections to the Higgs masses), or by chargino-stop loops (giving $\mathcal{O}\left(\alpha_{b} \alpha_{t}\right)$ corrections). Because the $\tan \beta$-enhanced contributions can be numerically relevant, an accurate determination of $h_{b}$ from the experimental value of the bottom mass requires a resummation of such effects to all orders in the perturbative expansion, as described in Refs. [29,30].

The leading effects are included in the effective Lagrangian formalism developed in Ref. [29]. Numerically this is by far the dominant part of the contributions from the sbottom sector (see also Refs. [26,27,31]). The dominant contributions arise from the loop-induced coupling of $H_{u}$ (the Higgs field that couples at the tree-level to up-type fermions only) to the down-type fermions. The effective Lagrangian is given by

$$
\begin{aligned}
& \mathcal{L}=\frac{g}{2 M_{W}} \frac{\bar{m}_{b}}{1+\Delta_{b}}\left[\quad \tan \beta A i \bar{b} \gamma_{5} b+\sqrt{2} V_{t b} \tan \beta H^{+} \bar{t}_{L} b_{R}\right. \\
& +\left(\frac{\sin \alpha}{\cos \beta}-\Delta_{b} \frac{\cos \alpha}{\sin \beta}\right) h \bar{b}_{L} b_{R} \\
& \left.-\left(\frac{\cos \alpha}{\cos \beta}+\Delta_{b} \frac{\sin \alpha}{\sin \beta}\right) H \bar{b}_{L} b_{R}\right]+ \text { h.c. . }
\end{aligned}
$$

Here $\bar{m}_{b}$ denotes the running bottom quark mass including SM QCD corrections. In the numerical evaluations obtained with FeynHiggs below we choose $\bar{m}_{b}=\bar{m}_{b}\left(m_{t}\right) \approx 2.97 \mathrm{GeV}$. The prefactor $1 /\left(1+\Delta_{b}\right)$ in eq. (14) arises from the resummation of the leading corrections to all orders. The additional terms $\sim \Delta_{b}$ in the $h \bar{b} b$ and $H \bar{b} b$ couplings arise from the mixing and coupling of the "other" Higgs boson, $H$ and $h$, respectively, to the $b$ quarks.

As explained above, the function $\Delta_{b}$ consists of two main contributions, an $\mathcal{O}\left(\alpha_{s}\right)$ correction from a sbottom-gluino loop and an $\mathcal{O}\left(\alpha_{t}\right)$ correction from a stop-higgsino loop. The explicit form of $\Delta_{b}$ in the limit of $M_{S} \gg m_{t}$ and $\tan \beta \gg 1$ reads [28]

$$
\Delta_{b}=\frac{2 \alpha_{s}}{3 \pi} m_{\tilde{g}} \mu \tan \beta \times I\left(m_{\tilde{b}_{1}}, m_{\tilde{b}_{2}}, m_{\tilde{g}}\right)+\frac{\alpha_{t}}{4 \pi} A_{t} \mu \tan \beta \times I\left(m_{\tilde{t}_{1}}, m_{\tilde{t}_{2}}, \mu\right) .
$$

The function $I$ is given by

$$
\begin{aligned}
I(a, b, c) & =\frac{1}{\left(a^{2}-b^{2}\right)\left(b^{2}-c^{2}\right)\left(a^{2}-c^{2}\right)}\left(a^{2} b^{2} \log \frac{a^{2}}{b^{2}}+b^{2} c^{2} \log \frac{b^{2}}{c^{2}}+c^{2} a^{2} \log \frac{c^{2}}{a^{2}}\right) \\
& \sim \frac{1}{\max \left(a^{2}, b^{2}, c^{2}\right)} .
\end{aligned}
$$


The large $\tilde{b}-\tilde{g}$ loops are resummed to all orders of $\left(\alpha_{s} \tan \beta\right)^{n}$ via the inclusion of $\Delta_{b}$ [28-30]. The leading electroweak contributions are taken into account via the second term in eq. (15).

For large values of $\tan \beta$ and the ratios of $\mu m_{\tilde{g}} / M_{\mathrm{SUSY}}^{2}$ and $\mu A_{t} / M_{\mathrm{SUSY}}^{2}$, the $\Delta_{b}$ correction can become very important. Considering positve values of $A_{t}$ and $m_{\tilde{g}}$, the sign of the $\Delta_{b}$ term is governed by the sign of $\mu$. Cancellations can occur if $A_{t}$ and $m_{\tilde{g}}$ have opposite signs. For $\mu, m_{\tilde{g}}, A_{t}>0$ the $\Delta_{b}$ correction is positive, leading to a suppression of the bottom Yukawa coupling. On the other hand, for negative values of $\Delta_{b}$, the bottom Yukawa coupling may be strongly enhanced and can even acquire non-perturbative values when $\Delta_{b} \rightarrow-1$.

\subsection{Impact on Higgs production and decay at large $\tan \beta$}

Higgs-boson production and decay processes at the Tevatron and the LHC can be affected by different kinds of large radiative corrections. The SM and MSSM corrections to the production channel $g g \rightarrow \phi$ have been calculated in Refs. [46,47], SM corrections to the $b \bar{b} \phi$ channel have been evaluated in Refs. [48-50]. Higgs decays to $b \bar{b}$ and to $\tau^{+} \tau^{-}$within the SM and MSSM have been evaluated including higher-order corrections in Refs. [16, 42,51]. Besides the process-specific corrections to the production and decay processes, large Higgsboson propagator contributions have an impact on the Higgs-boson couplings. For large $\tan \beta$ the supersymmetric radiative corrections to the bottom Yukawa coupling described above become particularly important [52]. Their main effect on the Higgs-boson production and decay processes can be understood from the way the leading contribution $\Delta_{b}$ enters. In the following we present simple analytic approximation formulae for the most relevant Higgs-boson production and decay processes. They are meant for illustration only so that the impact of the $\Delta_{b}$ corrections can easily be traced (for a discussion of possible enhancement factors for MSSM Higgs-boson production processes at the Tevatron and the LHC, see also Refs. $[53,54])$. In our numerical analysis below, we use the full result from FeynHiggs rather than the simple formulae presented in this section. No relevant modification to these results would be obtained using CPsuperH.

We begin with a simple approximate formula that represents well the MSSM parametric variation of the decay rate of the $\mathcal{C} \mathcal{P}$-odd Higgs boson in the large $\tan \beta$ regime. One should recall, for that purpose, that in this regime the $\mathcal{C} \mathcal{P}$-odd Higgs boson decays mainly into $\tau$-leptons and bottom-quarks, and that the partial decay widths are proportional to the square of the Yukawa couplings evaluated at an energy scale of about the Higgs boson mass. Moreover, for Higgs boson masses of the order of $100 \mathrm{GeV}$, the approximate relations $m_{b}\left(M_{A}\right)^{2} \simeq 9 \mathrm{GeV}^{2}$, and $m_{\tau}\left(M_{A}\right)^{2} \simeq 3 \mathrm{GeV}^{2}$ hold. Hence, since the number of colors is $N_{c}=3$, for heavy supersymmetric particles, with masses far above the Higgs boson mass scale, one has

$$
\begin{aligned}
\mathrm{BR}(A \rightarrow b \bar{b}) & \simeq \frac{9}{\left(1+\Delta_{b}\right)^{2}+9}, \\
\mathrm{BR}\left(A \rightarrow \tau^{+} \tau^{-}\right) & \simeq \frac{\left(1+\Delta_{b}\right)^{2}}{\left(1+\Delta_{b}\right)^{2}+9} .
\end{aligned}
$$

On the other hand, the production cross section for a $\mathcal{C P}$-odd Higgs boson produced in association with a pair of bottom quarks is proportional to the square of the bottom 
Yukawa coupling and therefore is proportional to $\tan ^{2} \beta /\left(1+\Delta_{b}\right)^{2}$. Also in the gluon fusion channel, the dominant contribution in the large $\tan \beta$ regime is governed by the bottom quark loops, and therefore is also proportional to the square of the bottom Yukawa coupling. Since the top-quark coupling is suppressed by either loop-corrections or inverse powers of $\tan \beta$, the leading top-quark correction arises from interference terms between the top-quark and bottom-quark loop diagrams. We have checked that these interference terms lead to corrections smaller than one percent (a few percent) for values of $\tan \beta \simeq 50$ (20). These corrections are small, of the order of other subleading corrections not included in our analysis, and lead to a very small modification of the current Tevatron limits (a small shift, smaller than $\Delta \tan \beta \sim 1$, in the limit on $\tan \beta$ ). They have been neglected in the CDF analysis of the $\sigma(p \bar{p} \rightarrow \phi) \times \operatorname{BR}\left(\phi \rightarrow \tau^{+} \tau^{-}\right)$process. We shall omit these corrections in the analytical formulae presented in this section and also in the numerical analysis below. However, including them would be straightforward (leading, as discussed above, to a very small modification of the allowed parameter space). Hence, the total production rate of bottom quarks and $\tau$ pairs mediated by the production of a $\mathcal{C} \mathcal{P}$-odd Higgs boson in the large $\tan \beta$ regime is approximately given by

$$
\begin{aligned}
\sigma(b \bar{b} A) \times \mathrm{BR}(A \rightarrow b \bar{b}) & \simeq \sigma(b \bar{b} A)_{\mathrm{SM}} \frac{\tan ^{2} \beta}{\left(1+\Delta_{b}\right)^{2}} \times \frac{9}{\left(1+\Delta_{b}\right)^{2}+9}, \\
\sigma(g g, b \bar{b} \rightarrow A) \times \mathrm{BR}\left(A \rightarrow \tau^{+} \tau^{-}\right) & \simeq \sigma(g g, b \bar{b} \rightarrow A)_{\mathrm{SM}} \frac{\tan ^{2} \beta}{\left(1+\Delta_{b}\right)^{2}+9},
\end{aligned}
$$

where $\sigma(b \bar{b} A)_{\mathrm{SM}}$ and $\sigma(g g, b \bar{b} \rightarrow A)_{\mathrm{SM}}$ denote the values of the corresponding SM Higgs boson production cross sections for a Higgs boson mass equal to $M_{A}$.

As a consequence, the $b \bar{b}$ production rate depends sensitively on $\Delta_{b}$ because of the factor $1 /\left(1+\Delta_{b}\right)^{2}$, while this leading dependence on $\Delta_{b}$ cancels out in the $\tau^{+} \tau^{-}$production rate. There is still a subdominant parametric dependence in the $\tau^{+} \tau^{-}$production rate on $\Delta_{b}$ that may lead to variations of a few tens of percent of the $\tau$-pair production rate (compared to variations of the rate by up to factors of a few in the case of bottom-quark pair production).

The formulae above apply, within a good approximation, also to the non-standard $\mathcal{C P}$ even Higgs boson in the large $\tan \beta$ regime. Indeed, unless the $\mathcal{C} \mathcal{P}$-odd Higgs mass is within a small regime of masses of about $m_{h}^{\max } \simeq 120-130 \mathrm{GeV}$, the mixing of the two $\mathcal{C} \mathcal{P}$-even Higgs bosons is small and, for $M_{A}>m_{h}^{\max }\left(M_{A}<m_{h}^{\max }\right), \cos \alpha \simeq \sin \beta(\sin \alpha \simeq-\sin \beta)$. In addition, this non-standard Higgs boson becomes degenerate in mass with the $\mathcal{C P}$-odd Higgs scalar. Therefore, the production and decay rates of $H(h)$ are governed by similar formulae as the ones presented above, leading to an approximate enhancement of a factor 2 of the production rates with respect to the ones that would be obtained in the case of the single production of the $\mathcal{C} \mathcal{P}$-odd Higgs boson as given in eqs. (19), (20). The same is true in the region where all three neutral Higgs bosons are approximately mass-degenerate, $m_{h} \simeq m_{H} \simeq M_{A}$. In this case the combined contribution of $h$ and $H$ to the production and decay rates approximately equals the contribution of the $\mathcal{C P}$-odd Higgs boson.

Besides the effects discussed above, additional radiative corrections can be important in the search for non-standard MSSM Higgs bosons. In particular, there are radiative corrections to the mass difference between $m_{H}$ (the non-SM like $\mathcal{C P}$-even Higgs boson) and $M_{A}$ [52]. If the two states are roughly mass-degenerate, one obtains a factor of 2 in the 
production rate, as outlined above. In the case of small mixing between the two $\mathcal{C} \mathcal{P}$-even states, the mass difference is approximately given by

$$
m_{H}^{2}-M_{A}^{2} \simeq-\frac{G_{F}}{4 \sqrt{2} \pi^{2}}\left[m_{t}^{4} \frac{\left(\mu A_{t}\right)^{2}}{M_{\mathrm{SUSY}}^{4}}+\frac{m_{b}^{4}}{\left(1+\Delta_{b}\right)^{4}} \frac{\left(\mu A_{b}\right)^{2}}{M_{\mathrm{SUSY}}^{4}}\right]
$$

where $G_{F}$ is the Fermi constant, and $m_{q}$ denotes the running quark masses. For large values of $\left(\mu A_{t}\right)$ and/or large values of $\left(\mu A_{b}\right)$ and $\tan \beta$, the mass difference becomes so large that the signals arising from the production of the $\mathcal{C P}$-even and the $\mathcal{C P}$-odd Higgs bosons can no longer simply be added, leading to a modification of the Higgs search sensitivity at the Tevatron and the LHC. A further important set of corrections are contributions from the sbottom sector giving rise to a large downward shift in the mass of the light $\mathcal{C P}$-even Higgs boson,

$$
\delta m_{h}^{2} \simeq-\frac{G_{F}}{4 \sqrt{2} \pi^{2}} \frac{m_{b}^{4}}{\left(1+\Delta_{b}\right)^{4}}\left(\frac{\mu}{M_{\mathrm{SUSY}}}\right)^{4} .
$$

For large values of $\mu$ and $\tan \beta$ these corrections can shift the prediction for $m_{h}$ below the experimental bound from LEP $[1,2]$. This can happen in particular for small mixing in the stop sector, for which the LEP bounds exclude a significant part of the parameter space. Finally, there are radiative corrections affecting the mixing of the two $\mathcal{C} \mathcal{P}$-even Higgs states that are not included in the above expressions. In particular, bottom-Yukawa induced corrections lead to an enhancement (suppression) of the mixing between the $\mathcal{C P}$ even Higgs bosons for large and negative (positive) values of $m_{b}^{4} /\left(1+\Delta_{b}\right)^{4} \mu^{3} A_{b} / M_{\mathrm{SUSY}}^{4}$. An enhancement of the mixing between $h$ and $H$ implies that the mass difference between $m_{H}$ and $M_{A}$ is pushed to larger values than those given in eq. (21) and that $m_{h}$ receives a further downward shift in addition to the correction in eq. (22).

We now turn to the production and decay processes of the charged Higgs boson. In the MSSM, the masses and couplings of the charged Higgs boson in the large $\tan \beta$ regime are closely related to the ones of the $\mathcal{C P}$-odd Higgs boson. The tree-level relation $M_{H^{ \pm}}^{2}=$ $M_{A}^{2}+M_{W}^{2}$ receives sizable corrections for large values of $\tan \beta, \mu, A_{t}$ and $A_{b}$,

$$
\begin{aligned}
M_{A}^{2} & \simeq M_{H^{ \pm}}^{2}-M_{W}^{2} \\
& +\frac{3 G_{F}}{8 \sqrt{2} \pi^{2}}\left[-\frac{m_{t}^{2} m_{b}^{2}}{\left(1+\Delta_{b}\right)^{2}}\left(4 \log \left(\frac{M_{S}^{2}}{m_{t}^{2}}\right)+2 A_{t b}\right)+\left(m_{t}^{4}+\frac{m_{b}^{4}}{\left(1+\Delta_{b}\right)^{4}}\right)\left(\frac{\mu}{M_{\mathrm{SUSY}}}\right)^{2}\right],
\end{aligned}
$$

with [18]

$$
A_{t b}=\frac{1}{6}\left[-\frac{6 \mu^{2}}{M_{\mathrm{SUSY}}^{2}}-\frac{\left(\mu^{2}-A_{b} A_{t}\right)^{2}}{M_{\mathrm{SUSY}}^{4}}+\frac{3\left(A_{t}+A_{b}\right)^{2}}{M_{\mathrm{SUSY}}^{2}}\right] .
$$

The coupling of the charged Higgs boson to a top and a bottom quark at large values of $\tan \beta$ is governed by the bottom Yukawa coupling and is therefore affected by the same $\Delta_{b}$ corrections that appear in the couplings of the non-standard neutral MSSM Higgs bosons [29].

The relevant channels for charged Higgs boson searches depend on its mass. For values of $M_{H^{ \pm}}$smaller than the top-quark mass, searches at hadron colliders concentrate on the possible emission of the charged Higgs boson from top-quark decays. In this case, for large 
values of $\tan \beta$, the charged Higgs decays predominantly into a $\tau$ lepton and a neutrino, i.e. one has to a good approximation

$$
\operatorname{BR}\left(H^{ \pm} \rightarrow \tau \nu_{\tau}\right) \approx 1
$$

The partial decay width of the top quark into a charged Higgs and a bottom quark is proportional to the square of the bottom Yukawa coupling and therefore scales with $\tan ^{2} \beta /\left(1+\Delta_{b}\right)^{2}$, see e.g. Ref. [29].

For values of the charged Higgs mass larger than $m_{t}$, instead, the most efficient production channel is the one of a charged Higgs associated with a top quark (mediated, for instance, by gluon-bottom fusion). In this case, the production cross section is proportional to the square of the bottom-quark Yukawa coupling. The branching ratio of the charged Higgs decay into a $\tau$ lepton and a neutrino is, apart from threshold corrections, governed by a similar formula as the branching ratio of the decay of the $\mathcal{C P}$-odd Higgs boson into $\tau$-pairs, namely

$$
\mathrm{BR}\left(H^{ \pm} \rightarrow \tau \nu_{\tau}\right) \simeq \frac{\left(1+\Delta_{b}\right)^{2}}{\left(1+\Delta_{b}\right)^{2}+9\left(1-r_{t}\right)^{2}}
$$

where the factor $\left(1-r_{t}\right)^{2}$ is associated with threshold corrections, and $r_{t}=m_{t}^{2} / M_{H^{ \pm}}^{2}$.

As mentioned above, our numerical analysis will be based on the complete expressions for the Higgs couplings rather than on the simple approximation formulae given in this section.

\section{Interpretation of cross section limits in MSSM scenarios}

\subsection{Limits at the Tevatron}

The D0 and CDF Collaborations have recently published cross section limits from the Higgs search at the Tevatron in the channel where at least three bottom quarks are identified in the final state $(b \bar{b} \phi, \phi \rightarrow b \bar{b})[7]$ and in the inclusive channel with $\tau^{+} \tau^{-}$final states $\left(p \bar{p} \rightarrow \phi \rightarrow \tau^{+} \tau^{-}\right)$[8]. The CDF Collaboration has also done analyses searching for a charged Higgs boson in top-quark decays [9]. For a Higgs boson with a mass of about $120 \mathrm{GeV}$, the D0 Collaboration excludes a cross section of about $30 \mathrm{pb}$ in the $b \bar{b} \phi, \phi \rightarrow b \bar{b}$ channel with a luminosity of $260 \mathrm{pb}^{-1}$ [7], and the CDF Collaboration excludes a cross section of about $15 \mathrm{pb}$ in the $p \bar{p} \rightarrow \phi \rightarrow \tau^{+} \tau^{-}$channel with a luminosity of $310 \mathrm{pb}^{-1}$ [8]. While the cross section for a SM Higgs boson is significantly below the above limits, a large enhancement of these cross sections is possible in the MSSM.

It is therefore of interest to interpret the cross section limits within the MSSM parameter space. Since the Higgs sector of the MSSM is characterised by two new parameters at lowest order, conventionally chosen as $M_{A}$ and $\tan \beta$, one usually displays the limits in the $M_{A^{-}}$ $\tan \beta$ plane (for $\mathcal{C} \mathcal{P}$-violating scenarios one normally chooses the $M_{H}^{ \pm}$-tan $\beta$ plane). As the whole structure of the MSSM enters via radiative corrections, the limits in the $M_{A}-\tan \beta$ plane depend on the other parameters of the model. One usually chooses certain benchmark scenarios to fix the other MSSM parameters [3,4]. In order to understand the physical 
meaning of the exclusion bounds in the $M_{A}-\tan \beta$ plane it is important to investigate how sensitively they depend on the values of the other MSSM parameters, i.e. on the choice of the benchmark scenarios.

\subsubsection{Limits from the process $b \bar{b} \phi, \phi \rightarrow b \bar{b}$}

The D0 Collaboration has presented the limits in the $M_{A}-\tan \beta$ plane obtained from the $b \bar{b} \phi, \phi \rightarrow b \bar{b}$ channel for the $m_{h}^{\max }$ and no-mixing scenarios as defined in Ref. [3]. The $m_{h}^{\max }$ scenario according to the definition of Ref. [3] reads

$$
\begin{aligned}
m_{t} & =174.3 \mathrm{GeV}, \\
M_{\mathrm{SUSY}} & =1000 \mathrm{GeV}, \\
\mu & =-200 \mathrm{GeV}, \\
M_{2} & =200 \mathrm{GeV}, \\
X_{t}^{\mathrm{OS}} & =2 M_{\mathrm{SUSY}}(\text { FD calculation }), \\
X_{t}^{\overline{\mathrm{MS}}} & =\sqrt{6} M_{\mathrm{SUSY}}(\text { RG calculation }) \\
A_{b} & =A_{t}, \\
m_{\tilde{g}} & =0.8 M_{\mathrm{SUSY}} .
\end{aligned}
$$

The no-mixing scenario defined in Ref. [3] differs from the $m_{h}^{\max }$ scenario only in

$$
X_{t}=0(\mathrm{FD} / \mathrm{RG} \text { calculation })
$$

The condition $A_{b}=A_{t}$ implies that the different mixing in the stop sector gives rise to a difference between the two scenarios also in the sbottom sector. The definition of the $m_{h}^{\max }$ and no-mixing scenarios given in Ref. [3] was later updated in Ref. [4], see the discussion below.

For their analysis, the D0 Collaboration has used the following approximate formula [7],

$$
\sigma(b \bar{b} \phi) \times \mathrm{BR}(\phi \rightarrow b \bar{b})=2 \sigma(b \bar{b} \phi)_{\mathrm{SM}} \frac{\tan ^{2} \beta}{\left(1+\Delta_{b}\right)^{2}} \times \frac{9}{\left(1+\Delta_{b}\right)^{2}+9}
$$

which follows from eq. (19) and the discussion in Sect. 2.3. The cross section $\sigma(b \bar{b} \phi)_{\mathrm{SM}}$ has been evaluated with the code of Ref. [48], while $\Delta_{b}$ has been calculated using CPsuperH [38]. From the discussion in Sect. 2.3] it follows that the choice of negative values of $\mu$ leads to an enhancement of the bottom Yukawa coupling and therefore to an enhancement of the signal cross section in eq. (29). For $\tan \beta=50$ the quantity $\Delta_{b}$ takes on the following values in the $m_{h}^{\max }$ and no-mixing scenarios as defined in eqs. (27), (28),

$$
\begin{array}{r}
m_{h}^{\max } \text { scenario, } \mu=-200 \mathrm{GeV}, \tan \beta=50: \Delta_{b}=-0.21 \\
\text { no-mixing scen., } \mu=-200 \mathrm{GeV}, M_{\mathrm{SUSY}}=1000 \mathrm{GeV}, \tan \beta=50: \Delta_{b}=-0.10
\end{array}
$$

While the $\mathcal{O}\left(\alpha_{s}\right)$ contribution to $\Delta_{b}$, see eq. (15), is practically the same in the two scenarios, the $\mathcal{O}\left(\alpha_{t}\right)$ contribution to $\Delta_{b}$ in the $m_{h}^{\max }$ scenario differs significantly from the one in the no-

mixing scenario. In the $m_{h}^{\max }$ scenario the $\mathcal{O}\left(\alpha_{t}\right)$ contribution to $\Delta_{b}$ is about as large as the 
$\mathcal{O}\left(\alpha_{s}\right)$ contribution. In the no-mixing scenario, on the other hand, the $\mathcal{O}\left(\alpha_{t}\right)$ contribution to $\Delta_{b}$ is very small, because $A_{t}$ is close to zero in this case. Reversing the sign of $\mu$ in eqs. (30), (31) reverses the sign of $\Delta_{b}$, leading therefore to a significant suppression of the signal cross section in eq. (29) for the same values of the other MSSM parameters.

The predictions for $b \bar{b} \phi, \phi \rightarrow b \bar{b}$ evaluated with FeynHiggs have been compared with the exclusion bound for $\sigma \times \mathrm{BR}$ as given in Ref. [7]. As mentioned above, in our analysis we use the full Higgs couplings obtained with FeynHiggs rather than the approximate formula given in eq. (29). Similar results would be obtained with CPsuperH.

The impact on the limits in the $M_{A}-\tan \beta$ plane from varying $\mu$ while keeping all other parameters fixed can easily be read off from eq. (29). For a given value of the $\mathcal{C P}$-odd mass and $\tan \beta$, the bound on $\sigma(b \bar{b} \phi) \times \mathrm{BR}(\phi \rightarrow b \bar{b})$ provides an upper bound on the bottomquark Yukawa coupling. The main effect therefore is that as $\mu$ varies, the bound on $\tan \beta$ also changes in such a way that the value of the bottom Yukawa coupling at the boundary line in the $M_{A}-\tan \beta$ plane remains the same.

The dependence of the limits in the $M_{A}-\tan \beta$ plane obtained from the process $b \bar{b} \phi, \phi \rightarrow b \bar{b}$ on the parameter $\mu$ is shown in Fig. 1. The limits for $\mu=-200 \mathrm{GeV}$ in the $m_{h}^{\max }$ and nomixing scenarios, corresponding to the limits presented by the D0 Collaboration in Ref. [7], are compared with the limits arising for different $\mu$ values, $\mu=+200, \pm 500, \pm 1000 \mathrm{GeV}$. Fig. 1illustrates that the effect of changing the sign of $\mu$ on the limits in the $M_{A}-\tan \beta$ plane obtained from the process $b \bar{b} \phi, \phi \rightarrow b \bar{b}$ is quite dramatic. In the $m_{h}^{\max }$ scenario the exclusion bound degrades from about $\tan \beta=50$ for $M_{A}=90 \mathrm{GeV}$ in the case of $\mu=-200 \mathrm{GeV}$ to about $\tan \beta=90$ for $M_{A}=90 \mathrm{GeV}$ in the case of $\mu=+200 \mathrm{GeV}$. We extend our plots to values of $\tan \beta$ much larger than 50 mainly for illustration purposes; the region $\tan \beta \gg 50$ in the MSSM is theoretically disfavoured, if one demands that the values of the bottom and $\tau$ Yukawa couplings remain in the perturbative regime up to energies of the order of the unification scale. The situation for the bottom-Yukawa coupling can be ameliorated for large positive values of $\mu$ due to the $\Delta_{b}$ corrections. The curves for $\mu=+500,+1000 \mathrm{GeV}$ do not appear in the plot for the $m_{h}^{\max }$ scenario, since for these $\mu$ values there is no $\tan \beta$ exclusion below $\tan \beta=130$ for any value of $M_{A}$. On the other hand, the large negative values of $\mu$ shown in Fig. 1 $\mu=-500,-1000 \mathrm{GeV}$, lead to an even stronger enhancement of the signal cross section than for $\mu=-200 \mathrm{GeV}$ and, accordingly, to an improved reach in $\tan \beta$. It should be noted that for $\mu=-500,-1000 \mathrm{GeV}$ the bottom Yukawa coupling becomes so large for $\tan \beta \gg 50$ that a perturbative treatment would no longer be reliable in this region.

In the no-mixing scenario, where the absolute value of $\Delta_{b}$ is smaller, the exclusion bound is shifted from about $\tan \beta=55$ for $M_{A}=90 \mathrm{GeV}$ in the case of $\mu=-200 \mathrm{GeV}$ to about $\tan \beta=75$ for $M_{A}=90 \mathrm{GeV}$ in the case of $\mu=+200 \mathrm{GeV}$. For $\mu=+500 \mathrm{GeV}$, no excluded region can be established below $\tan \beta=100$. As above, large negative values of $\mu$, i.e. $\mu=-500,-1000 \mathrm{GeV}$, result in an improved reach in $\tan \beta$ compared to the value $\mu=-200 \mathrm{GeV}$ chosen by the D0 Collaboration.

The variation with the sign and absolute value of the $\mu$-parameter shows the strong dependence of the limits in the $M_{A}-\tan \beta$ plane on the strength of the bottom-quark Yukawa coupling and hence on the supersymmetric parameter space. The sensitive dependence of the process $b \bar{b} \phi, \phi \rightarrow b \bar{b}$ on the bottom-quark Yukawa coupling is not specific to the particular benchmark scenarios considered here. Keeping the ratio of $\mu m_{\tilde{g}} / M_{\text {SUSY }}^{2}$ fixed but varying $\mu$ 

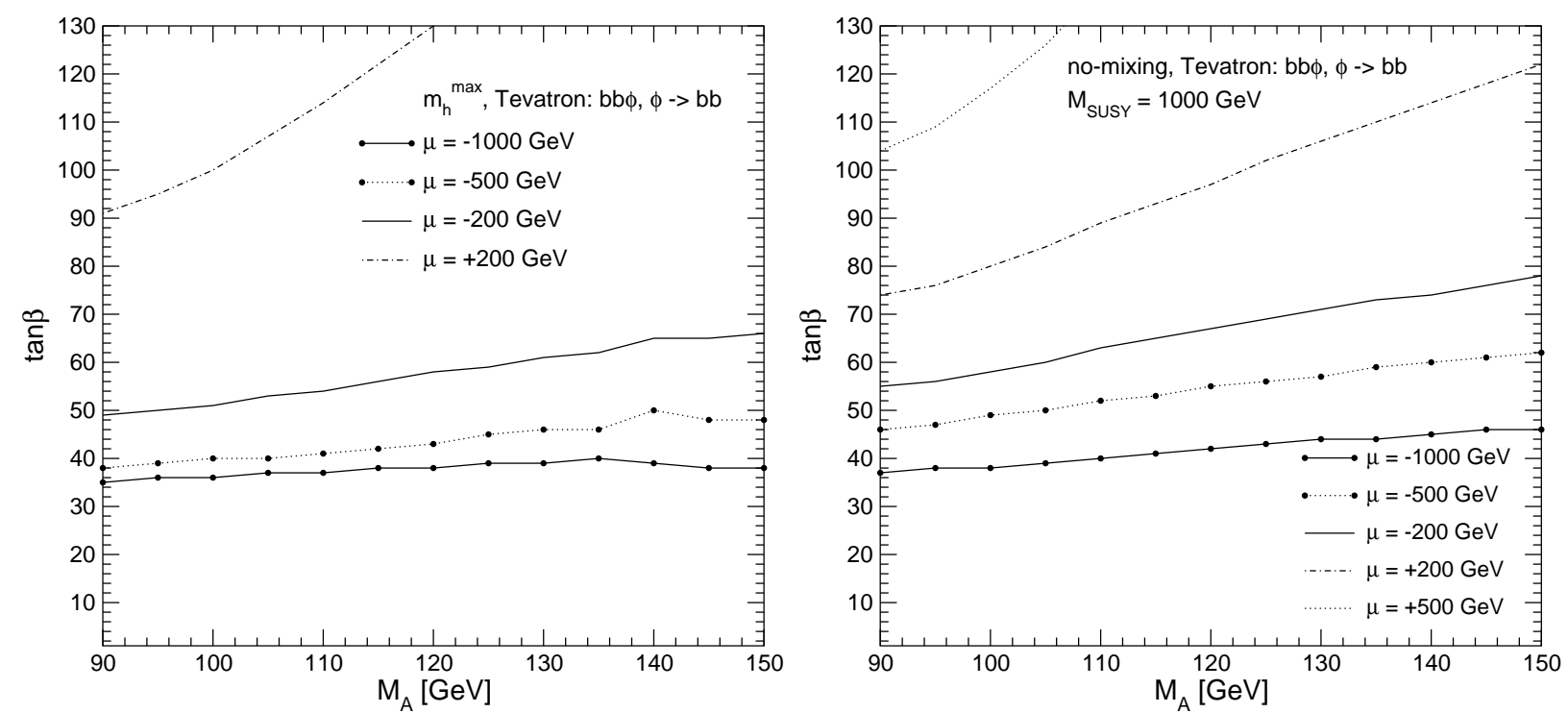

Figure 1: Change in the limits obtained from the $b \bar{b} \phi, \phi \rightarrow b \bar{b}$ channel in the $m_{h}^{\max }$ (left) and no-mixing (right) benchmark scenarios for different values of $\mu$. The value $\mu=-200 \mathrm{GeV}$ was chosen by the D0 Collaboration in Ref. [7]. The other curves indicate the corresponding limits for $\mu=+200, \pm 500, \pm 1000 \mathrm{GeV}$. The curves for $\mu=+500,+1000 \mathrm{GeV}$ $(\mu=+1000 \mathrm{GeV})$ do not appear in the left (right) plot for the $m_{h}^{\max }$ (no-mixing) scenario, since for these $\mu$ values there is no $\tan \beta$ exclusion below $\tan \beta=130$ for any value of $M_{A}$.

and $m_{\tilde{g}}$ independently will lead to similar results as those shown here. A scenario where large compensations are possible between the two contributions entering $\Delta_{b}$, see eq. (15), will be discussed below. Scenarios with different values of the other supersymmetric parameters (besides the ones entering $\Delta_{b}$ ) will reproduce a similar behaviour as those discussed here.

In Ref. [4] the definition of the $m_{h}^{\max }$ and no-mixing scenarios given in Ref. [3] has been updated, and the "small $\alpha_{\text {eff" }}$ scenario and the "gluophobic Higgs scenario" have been proposed as additional scenarios for the search for the light $\mathcal{C} \mathcal{P}$-even Higgs boson at the Tevatron and the LHC. The sign of $\mu$ in the $m_{h}^{\max }$ and no-mixing scenarios has been reversed to $\mu=+200 \mathrm{GeV}$ in Ref. [4]. This leads typically to a better agreement with the constraints from $(g-2)_{\mu}$. Furthermore, the value of $M_{\mathrm{SUSY}}$ in the no-mixing scenario was increased from $1000 \mathrm{GeV}$ [3] to $2000 \mathrm{GeV}$ in order to ensure that most of the parameter space of this scenario is in accordance with the LEP exclusion bounds $[1,2]$.

Another scenario defined in Ref. [4] is the "constrained- $m_{h}^{\max }$ " scenario. It differs from the $m_{h}^{\max }$ scenario as specified in Ref. [4] by the reversed sign of $X_{t}$,

$$
\begin{aligned}
X_{t}^{\mathrm{OS}} & =-2 M_{\mathrm{SUSY}}(\mathrm{FD} \text { calculation }), \\
X_{t}^{\overline{\mathrm{MS}}} & =-\sqrt{6} M_{\mathrm{SUSY}}(\mathrm{RG} \text { calculation }), \\
\mu & =+200 \mathrm{GeV} .
\end{aligned}
$$

For small $M_{A}$ and minimal flavor violation this results in better agreement with the constraints from $\operatorname{BR}(b \rightarrow s \gamma)$. For large $\tan \beta$ one has $A_{t} \approx X_{t}$, thus $A_{t}$ and $m_{\tilde{g}}$ have opposite signs. This can lead to cancellations in the two contributions entering $\Delta_{b}$, see eq. (15). In 
contrast to the $m_{h}^{\max }$ scenario, where the two contributions entering $\Delta_{b}$ add up, see eq. (30), the constrained- $m_{h}^{\max }$ scenario typically yields relatively small values of $\Delta_{b}$ and therefore a correspondingly smaller effect on the relation between the bottom-quark mass and the bottom Yukawa coupling, e.g.

$$
\text { constrained- } m_{h}^{\max } \text { scenario, } \mu=+200 \mathrm{GeV}, \tan \beta=50: \Delta_{b}=-0.001 \text {. }
$$

For large values of $|\mu|$ the compensations between the two terms entering $\Delta_{b}$ are less efficient, since the function $I$ in the second term of eq. (30) scales like $1 / \mu^{2}$ for large $|\mu|$.

We now study the impact of the benchmark definitions of Ref. [4] on the limits in the $M_{A}-\tan \beta$ plane arising from the $b \bar{b} \phi, \phi \rightarrow b \bar{b}$ channel. The left plot in Fig. 2 shows the effect of changing $M_{\mathrm{SUSY}}=1000 \mathrm{GeV}$ to $M_{\mathrm{SUSY}}=2000 \mathrm{GeV}$ in the no-mixing scenario for $\mu= \pm 200 \mathrm{GeV}$. Due to the heavier scalar bottoms in the case of $M_{\mathrm{SUSY}}=2000 \mathrm{GeV}$ the effect of the $\Delta_{b}$ corrections is suppressed as compared to the benchmark definition in Ref. [3]. This leads to a shift of the limits in the $M_{A}-\tan \beta$ plane of about $\Delta \tan \beta=5-10$ for a given value of $M_{A}$. The right plot of Fig. 2] shows for $M_{\mathrm{SUSY}}=2000 \mathrm{GeV}$ the variation of the limits with $\mu$. In this case even for $\mu=+1000 \mathrm{GeV}$ a $\tan \beta$ exclusion limit can be established below $\tan \beta=130$, in contrast to the scenario with $M_{\mathrm{SUSY}}=1000 \mathrm{GeV}$, see Fig. 11.
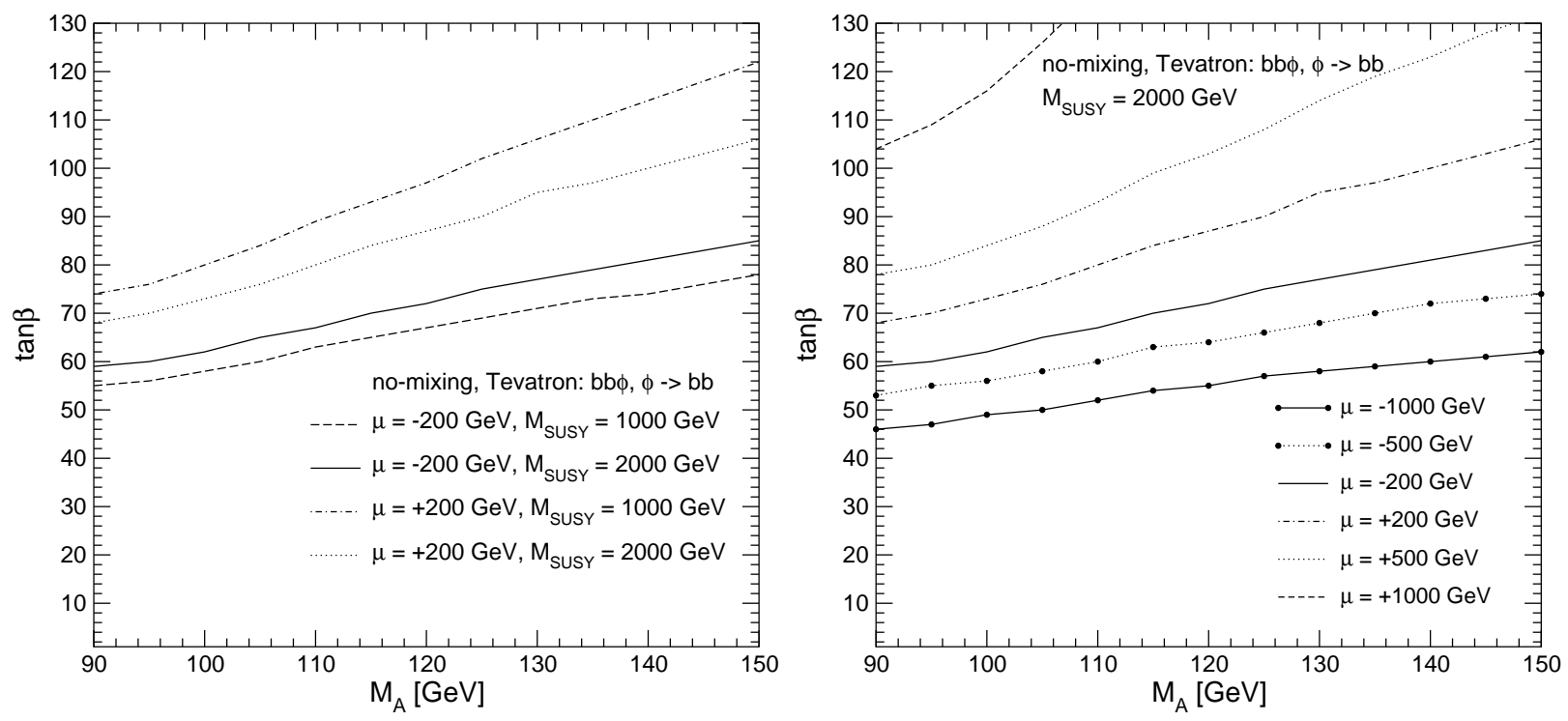

Figure 2: Variation of the limits obtained from the $b \bar{b} \phi, \phi \rightarrow b \bar{b}$ channel in the nomixing scenario for different values of $M_{\mathrm{SUSY}}$ and $\mu$. The left plot shows the results for $M_{\mathrm{SUSY}}=1000,2000 \mathrm{GeV}$ and $\mu= \pm 200 \mathrm{GeV}$, while in the right plot the results for $M_{\mathrm{SUSY}}=2000 \mathrm{GeV}$ and $\mu= \pm 200, \pm 500, \pm 1000 \mathrm{GeV}$ are given.

The results in the constrained- $m_{h}^{\max }$ scenario are displayed in Fig. 3 (left). The results are shown for $\mu= \pm 200, \pm 500 \mathrm{GeV}$. As expected from the discussion above, the obtained limits are relatively stable against the variation of $\mu$. For $\mu=+500(-500) \mathrm{GeV}$ the $\tan \beta$ limit is significantly weaker (stronger) than for smaller values of $|\mu|$ as a consequence of the less efficient cancellation of the two contributions to $\Delta_{b}$ discussed above. Nevertheless, the limits obtained for $|\mu| \leq 500 \mathrm{GeV}$ are weaker than those for the $m_{h}^{\max }$ scenario with negative 
$\mu$, but stronger than those for positive $\mu$. The curve for $\mu=+1000 \mathrm{GeV}$ is not shown in the plot, since for this value there is no $\tan \beta$ exclusion below $\tan \beta=130$ for any value of $M_{A}$. For $\mu=-1000 \mathrm{GeV}$, on the other hand, the radiative corrections lead to a large mass splitting between the $\mathcal{C P}$-odd and $\mathcal{C P}$-even Higgs boson masses so that the approximation of adding the two signal cross sections is no longer valid, see the discussion in Sect. 2.3. A more detailed study would be necessary to incorporate also the case of larger Higgs boson mass splittings.
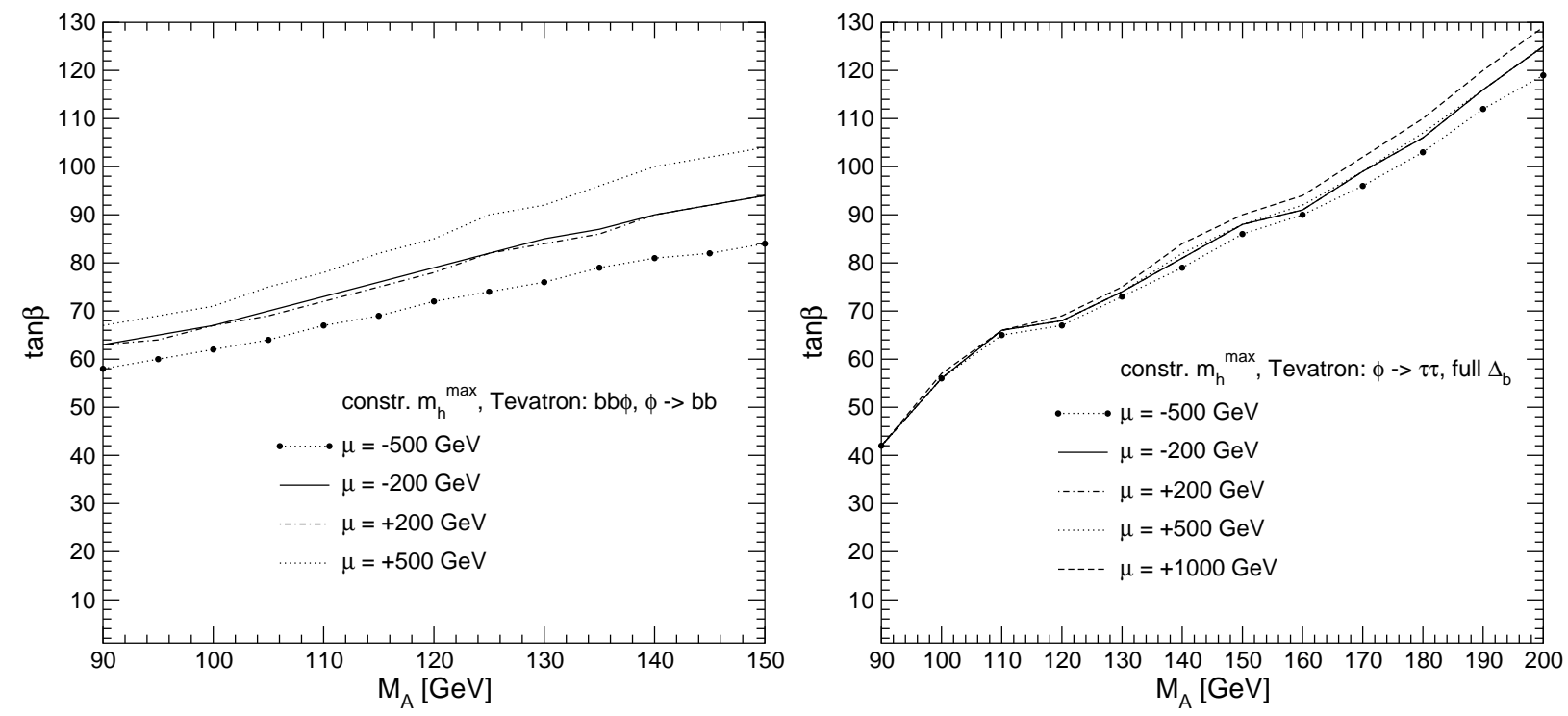

Figure 3: Left: Variation of the limits obtained from the $b \bar{b} \phi, \phi \rightarrow b \bar{b}$ channel in the constrained $-m_{h}^{\max }$ scenario for different values of $\mu$. Right: Variation of the limits obtained from the $p \bar{p} \rightarrow \phi \rightarrow \tau^{+} \tau^{-}$channel in the constrained $-m_{h}^{\max }$ scenario for different values of $\mu$.

\subsubsection{Limits from the process $p \bar{p} \rightarrow \phi \rightarrow \tau^{+} \tau^{-}$}

The limits obtained from the $p \bar{p} \rightarrow \phi \rightarrow \tau^{+} \tau^{-}$channel by the CDF Collaboration were presented in the $M_{A}-\tan \beta$ plane for the $m_{h}^{\max }$ and no-mixing scenarios as defined in Ref. [4] and employing two values of the $\mu$ parameter, $\mu= \pm 200 \mathrm{GeV}$. According to the discussion in Sect. 2.3. the limits obtained from the $p \bar{p} \rightarrow \phi \rightarrow \tau^{+} \tau^{-}$channel are expected to show a weaker dependence on the sign and absolute value of $\mu$ than the limits arising from the $b \bar{b} \phi, \phi \rightarrow b \bar{b}$ channel. On the other hand, for large values of $\tan \beta$ and negative values of $\mu$, the large corrections to the bottom Yukawa coupling discussed above can invalidate a perturbative treatment for this channel.

The MSSM prediction for $\sigma(p \bar{p} \rightarrow \phi) \times \mathrm{BR}\left(\phi \rightarrow \tau^{+} \tau^{-}\right)$as a function of $\tan \beta$ has been evaluated by the CDF collaboration using the HIGLU program [55] for the gluon fusion channel. The prediction for the $b \bar{b} \rightarrow \phi+X$ channel was obtained from the NNLO result in the SM from Ref. [50], and $[\sigma \times \mathrm{BR}]_{\mathrm{MSSM}} /[\sigma \times \mathrm{BR}]_{\mathrm{SM}}$ was calculated with the FeynHiggs program [34-37]. While the full $\Delta_{b}$ correction to the bottom Yukawa correction was taken into account in the $b \bar{b} \rightarrow \phi+X$ production channel and the $\phi \rightarrow \tau^{+} \tau^{-}$branching ratios, 
the public version of the HIGLU program [55] does not include the $\Delta_{b}$ correction for the bottom Yukawa coupling entering the bottom loop contribution to the $g g \rightarrow \phi$ production process. In order to treat the two contributing production processes in a uniform way, the $\Delta_{b}$ correction should be included (taking into account the $\mathcal{O}\left(\alpha_{s}\right)$ and the $\mathcal{O}\left(\alpha_{t}\right)$ parts, see eq. (15)) in the $g g \rightarrow \phi$ production process calculation. For the large value of $M_{\mathrm{SUSY}}$ chosen in the $m_{h}^{\max }$ and no-mixing benchmark scenarios other higher-order contributions involving sbottoms and stops can be neglected (these effects are small provided $M_{\mathrm{SUSY}} \gtrsim 500 \mathrm{GeV}$ ).

We therefore begin the investigation of the $p \bar{p} \rightarrow \phi \rightarrow \tau^{+} \tau^{-}$channel by analyzing the impact of including or omitting the $\Delta_{b}$ correction in the $g g \rightarrow \phi$ production process. In order to get a qualitative understanding of the variation of the limits on $\tan \beta$ for a given value of $M_{A}$ induced by the inclusion of the $\Delta_{b}$ corrections in the gluon fusion channel, it is again useful to employ the simple approximate formulae given in Sect. 2.3. As discussed above, the production cross sections may be approximately obtained from the SM ones by including a simple rescaling by a factor $\tan ^{2} \beta /\left(1+\Delta_{b}\right)^{2}$. Hence, defining $\sigma_{b}$ and $\sigma_{g}$ as the SM cross sections for the $b$-quark associated and gluon fusion production of Higgs bosons, respectively, we get

$$
\begin{aligned}
\sigma(p \bar{p} \rightarrow \phi) \times\left.\mathrm{BR}\left(\phi \rightarrow \tau^{+} \tau^{-}\right)\right|_{\text {full } \Delta_{b}} & \simeq\left(\sigma_{b}+\sigma_{g}\right) \times \frac{\tan ^{2} \beta}{\left(1+\Delta_{b}\right)^{2}+9} \\
\sigma(p \bar{p} \rightarrow \phi) \times\left.\mathrm{BR}\left(\phi \rightarrow \tau^{+} \tau^{-}\right)\right|_{\text {partial } \Delta_{b}} & \simeq\left(\sigma_{b}+\sigma_{g}\left(1+\Delta_{b}\right)^{2}\right) \times \frac{\tan ^{2} \beta}{\left(1+\Delta_{b}\right)^{2}+9},
\end{aligned}
$$

where "full $\Delta_{b}$ " denotes the case where the $\Delta_{b}$ correction is incorporated in both the $b$ quark associated and the gluon fusion production processes (and the $\phi \rightarrow \tau^{+} \tau^{-}$branching ratio), while "partial $\Delta_{b}$ " denotes the case where the $\Delta_{b}$ correction is omitted in the $g g \rightarrow \phi$ production process. The expression above shows that, for positive values of $\mu$, for which $\Delta_{b}>0$, the omission of the correction to the $g g \rightarrow \phi$ process leads to an enhancement of the total production cross section with respect to the value obtained when these corrections are included. For negative values of $\mu$, instead, the situation is reversed.

The cross section for the $g g \rightarrow \phi$ production process including the $\Delta_{b}$ correction can be obtained by a simple rescaling of the HIGLU result for the SM. The cross section for the SM production rate involving the $b$-quark loop alone is rescaled with $\Gamma(h \rightarrow b \bar{b})_{\mathrm{MSSM}} / \Gamma(h \rightarrow$ $b \bar{b})_{\mathrm{SM}}$, where $\Delta_{b}$ enters the calculation of $\Gamma(h \rightarrow b \bar{b})_{\mathrm{MSSM}}$ (SM-QCD corrections to the $b$ quark mass factorize and drop out in this ratio). As stressed above, it has been checked that the $t$-quark loop gives only a negligible contribution in the MSSM for $\tan \beta \gtrsim 20$. The loops involving scalar tops and bottoms, beyond those included in the $\Delta_{b}$ corrections, also give small contributions due the heavy scalar masses in the benchmark scenarios.

The comparison of the "partial $\Delta_{b}$ " and the "full $\Delta_{b}$ " results is shown in Fig. 4. The evaluation of $\sigma \times \mathrm{BR}$ in the MSSM has been performed by using FeynHiggs for rescaling the HIGLU results for the $g g \rightarrow \phi$ production cross sections and the SM results for the $b \bar{b} \rightarrow \phi+X$ channel [50] by the appropriate MSSM correction factor, as outlined above. The impact of the additional contribution can be read off from Fig. 4 by comparing the results where the $\Delta_{b}$ corrections are omitted in the $g g \rightarrow \phi$ production cross sections ("partial $\Delta_{b}$ ") with the results where the $\Delta_{b}$ corrections have been taken into account everywhere in the production and decay processes ("full $\Delta_{b}$ "). The effect on the exclusion bounds in the $M_{A}$-tan $\beta$ plane is seen to be quite significant. While in the case where the $\Delta_{b}$ corrections are neglected in the 
$g g \rightarrow \phi$ production cross sections the strongest exclusion bounds are obtained for positive values of $\mu$, the inclusion of the $\Delta_{b}$ corrections reverses this situation. As explained above, the inclusion of the $\Delta_{b}$ corrections to the $g g \rightarrow \phi$ production process leads to a larger cross section (and correspondingly to a stronger $\tan \beta$ bound) in the case of negative $\mu$, while the cross section is suppressed for positive values of $\mu$. The corresponding shifts of the exclusion limits in the $M_{A}-\tan \beta$ plane amount up to $\Delta \tan \beta \sim 10$ for the $m_{h}^{\max }$ scenario. In the nomixing sceanrio (defined according to Ref. [4]) the effect is less pronounced because of the smaller numerical value of $\Delta_{b}$, giving rise to shifts in the exclusion limits up to $\Delta \tan \beta \sim 5$.
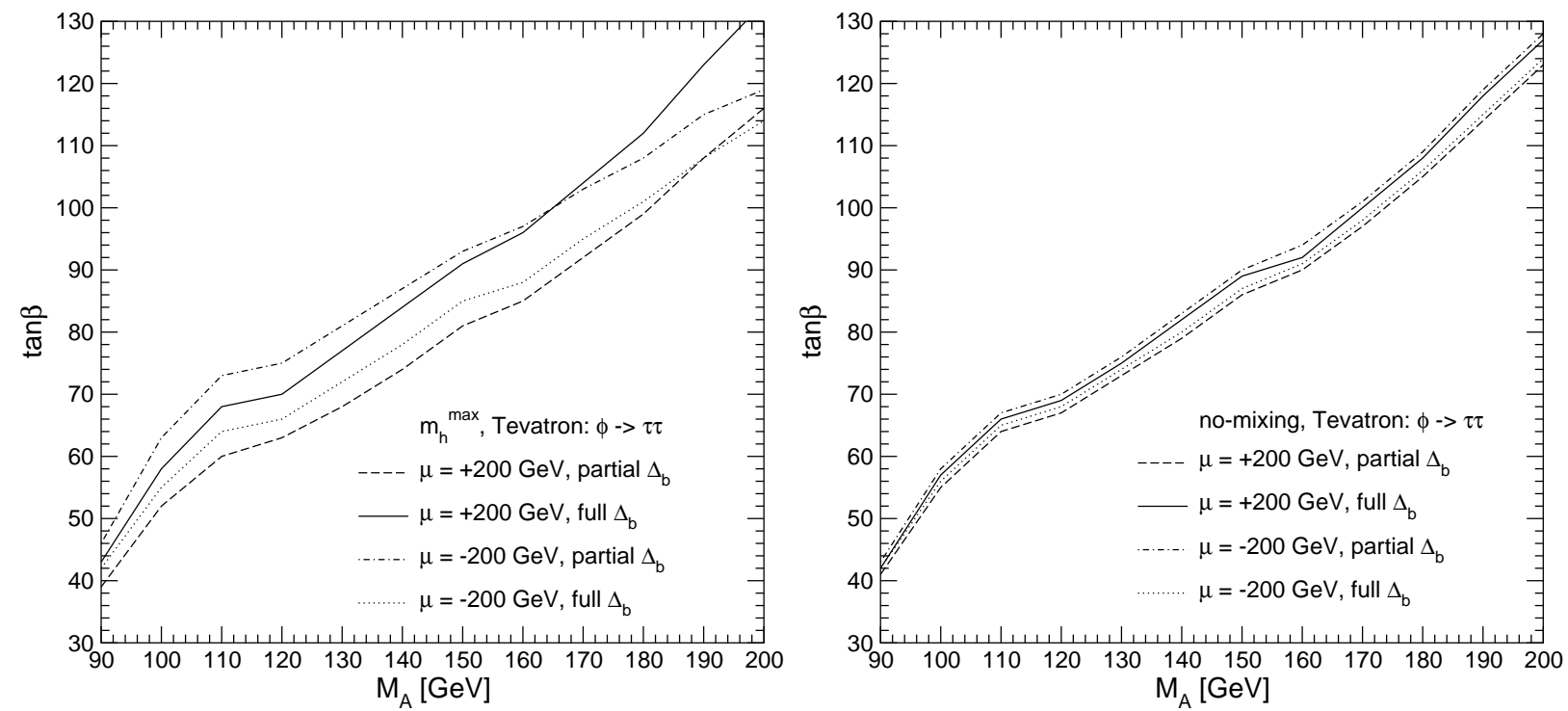

Figure 4: Impact of including or omitting the $\Delta_{b}$ correction in the $g g \rightarrow \phi$ production process on the limits obtained from the $p \bar{p} \rightarrow \phi, \phi \rightarrow \tau^{+} \tau^{-}$channel. The results are shown for $\mu= \pm 200 \mathrm{GeV}$ in the $m_{h}^{\max }$ (left) and no-mixing (right) benchmark scenarios [4].

Following our analysis, the CDF Collaboration has adopted the prescription outlined above for incorporating the $\Delta_{b}$ correction into the $g g \rightarrow \phi$ production process. The limits given in Ref. [8] are based on the MSSM prediction where the $\Delta_{b}$ correction is included everywhere in the production and decay processes (see e.g. Ref. [56] for a previous analysis).

We next turn to the discussion of the sensitivity of the limits obtained from the $p \bar{p} \rightarrow$ $\phi \rightarrow \tau^{+} \tau^{-}$channel (including the $\Delta_{b}$ correction in all production and decay processes) on the sign and absolute value of $\mu$. As discussed above, similar variations in the exclusion limits will occur if the absolute values of $\mu, m_{\tilde{g}}, A_{t}$ and $M_{\mathrm{SUSY}}$ are varied, while keeping the ratios appearing in $\Delta_{b}$ constant. The results are given in Fig. 5 for the $m_{h}^{\max }$ scenario (left) and the no-mixing scenario (right). In the $m_{h}^{\max }$ scenario we find a sizable dependence of the $\tan \beta$ bounds on the sign and absolute value of $\mu{ }^{2}$ The effect grows with $M_{A}$ and, for the range of parameters explored in Fig. [5] leads to a variation of the $\tan \beta$ bound larger than

\footnotetext{
${ }^{2}$ For $\mu=-300 \mathrm{GeV}$ the curve stops at around $\tan \beta=75$ because the bottom Yukawa coupling becomes very large, leading to instabilities in the calculation of the Higgs properties. For the same reason, even more negative values of $\mu$ are not considered here.
} 
$\Delta \tan \beta \sim 30$. In the no-mixing scenario the effect is again smaller, but it can still lead to a variation of the $\tan \beta$ bounds by as much as $\Delta \tan \beta \sim 10$.
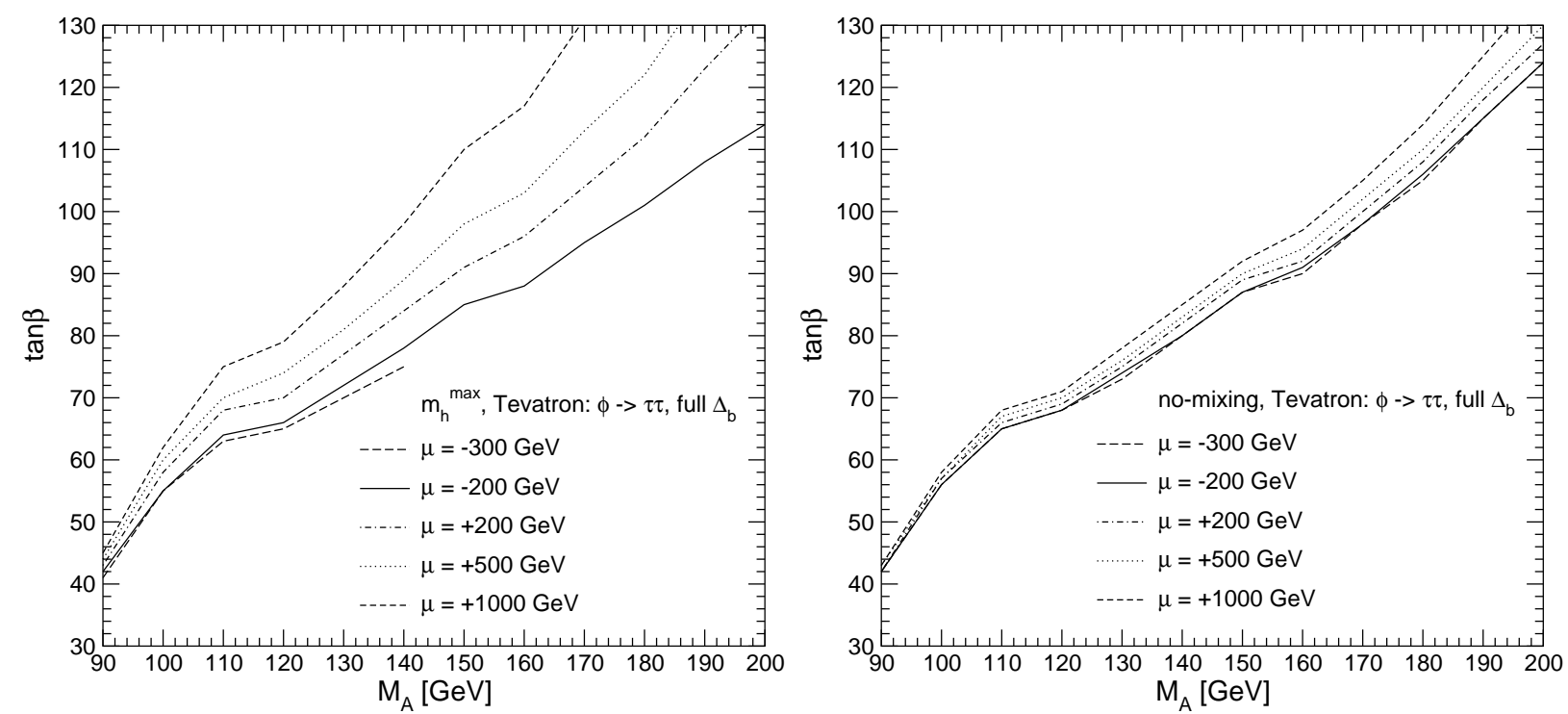

Figure 5: Variation of the limits obtained from the $p \bar{p} \rightarrow \phi \rightarrow \tau^{+} \tau^{-}$channel at the Tevatron in the $m_{h}^{\max }$ (left) and no-mixing (right) benchmark scenarios for different values of $\mu$.

The results obtained in the constrained $-m_{h}^{\max }$ scenario are shown in Fig. 3 (right). As expected, the exclusion limits in this scenario are very robust with respect to varying $\mu$. All values of $\mu$ result practically in the same $\tan \beta$ exclusion bounds. The lines not visible in the plot are actually covered by a line of another $\mu$ value. For $\mu=-1000 \mathrm{GeV}$, the radiative corrections lead to a large mass splitting between the $\mathcal{C P}$-odd and $\mathcal{C P}$-even Higgs boson masses, see the discussion above.

\subsubsection{Limits from the process $p \bar{p} \rightarrow t \bar{t} \rightarrow H^{ \pm} W^{\mp} b \bar{b}, H^{ \pm} \rightarrow \tau \nu_{\tau}$}

For the charged Higgs search channel at the Tevatron [9], $p \bar{p} \rightarrow t \bar{t} \rightarrow H^{ \pm} W^{\mp} b \bar{b}, H^{ \pm} \rightarrow \tau \nu_{\tau}$ the variation of the cross section with $\mu$ is driven by the impact of the $\Delta_{b}$ correction on $\operatorname{BR}\left(t \rightarrow H^{ \pm} b\right)[29]$. The decay width $\Gamma\left(t \rightarrow H^{ \pm} b\right)$ is proportional to $\tan ^{2} \beta /\left(1+\Delta_{b}\right)^{2}$, leading to an expression for the branching ratio in analogy to eq. (17). Accordingly, a positive $\Delta_{b}$ leads to a suppression of $\operatorname{BR}\left(t \rightarrow H^{ \pm} b\right)$, while a negative $\Delta_{b}$ leads to an enhancement.

For a fixed value of $M_{H^{ \pm}}$, the value of $M_{A}$ is driven to rather small values because of the tree-level relation $M_{A}^{2}=M_{H^{ \pm}}^{2}-M_{W}^{2}$. For large values of $\tan \beta, A_{t}$ and $A_{b}$ this effect is further enhanced by the higher-order corrections in eq. (23). Consequently, in the region of small $M_{H^{ \pm}}$and large $\tan \beta$ currently probed at the Tevatron [9] the corresponding $M_{A}$ values tend to be further reduced with respect to the already small tree-level values ${ }^{3}$ and are in general below the LEP exclusion bound [2]. Therefore, this channel at present is less

\footnotetext{
${ }^{3}$ This effect is avoided if the parameters $A_{t}, A_{b}$ and $\mu$ are such that $A_{t b}$ in eq. (24) becomes sufficiently large and negative. This can be realized, for instance, for values of $M_{H^{ \pm}}>120 \mathrm{GeV}$ and $A_{t}=-A_{b}$ in the constrained- $m_{h}^{\max }$ scenario, for small values of $\mu$.
} 
relevant for obtaining exclusion limits in the $M_{A}-\tan \beta$ plane than the neutral Higgs-boson search channels discussed above. It is expected to become more competitive, however, with increasing luminosity collected in Run II of the Tevatron.

\subsection{Prospects for Higgs sensitivities at the LHC}

The most sensitive channels for detecting heavy MSSM Higgs bosons at the LHC are the channel $p p \rightarrow H / A+X, H / A \rightarrow \tau^{+} \tau^{-}$(making use of different decay modes of the two $\tau$ leptons) and the channel $t H^{ \pm}, H^{ \pm} \rightarrow \tau \nu_{\tau}$ (for $M_{H^{ \pm}} \geq m_{t}$ ) $[57,58]$. We consider here the parameter region $M_{A} \gg M_{Z}$, for which the heavy states $H, A$ are widely separated in mass from the light $\mathcal{C P}$-even Higgs boson $h$. Here and in the following we do not discuss search channels where the heavy Higgs bosons decay into supersymmetric particles, which depend very sensitively on the model parameters [58-60], but we will comment below on how these decays can affect the searches with bottom-quarks and $\tau$-leptons in the final state.

\subsubsection{Discovery region for the process $p p \rightarrow H / A+X, H / A \rightarrow \tau^{+} \tau^{-}$}

To be specific, we concentrate in this section on the analysis carried out by the CMS Collaboration $[58,61]$. Similar results for this channel have also been obtained by the ATLAS Collaboration [57,62]. In order to rescale the SM cross sections and branching ratios, the CMS Collaboration has used for the branching ratios the HDECAY program [63] and for the production cross sections the HIGLU program [55] $(g g \rightarrow H / A)$ and the HQQ program [64] $(g g \rightarrow b \bar{b} H)$. In the HDECAY program the $\Delta_{b}$ corrections are partially included for the decays of the neutral Higgs bosons (only the $\mathcal{O}\left(\alpha_{s}\right)$ contribution to $\Delta_{b}$ is included, see eq. (15)). The HIGLU program (see also the discussion in Sect. 3.1.2) and HQQ, on the other hand, do not take into account the corrections to the bottom Yukawa coupling. ${ }^{4}$ The prospective $5 \sigma$ discovery contours for CMS (corresponding to the upper bound of the LHC "wedge" region, where only the light $\mathcal{C} \mathcal{P}$-even Higgs boson may be observed at the LHC) have been presented in Refs. $[58,61]$ in the $M_{A}-\tan \beta$ plane, for an integrated luminosity of $30 \mathrm{fb}^{-1}$ and $60 \mathrm{fb}^{-1}$. The results were presented in the $m_{h}^{\max }$ scenario and for different $\mu$ values, $\mu=-200,+300,+500 \mathrm{GeV}$. It should be noted that decays of heavy Higgs bosons into charginos and neutralinos open up for small enough values of the soft supersymmetrybreaking parameters $M_{2}$ and $\mu$. Indeed, the results presented in Refs. $[58,61]$ show a degradation of the discovery reach in the $M_{A}-\tan \beta$ plane for smaller absolute values of $\mu$, which is due to an enhanced branching ratio of $H, A$ into supersymmetric particles, and accordingly a reduced branching ratio into $\tau$ pairs.

We shall now study the impact of including the $\Delta_{b}$ corrections into the production cross sections and branching ratios for different values of $\mu$. The inclusion of the $\Delta_{b}$ corrections leads to a modification of the dependence of the production cross section on $\tan \beta$, as well as of the branching ratios of the Higgs boson decays into $\tau^{+} \tau^{-}$. For a fixed value of $M_{A}$, the results obtained by the CMS Collaboration for the discovery region in $\tan \beta$ can be interpreted in terms of a cross section limit using the approximation of rescaling the SM rate

\footnotetext{
${ }^{4}$ Since HQQ is a leading-order program, non-negligible changes can also be expected from SM-QCD type higher-order corrections.
} 
for the $p p \rightarrow H+X, H \rightarrow \tau^{+} \tau^{-}$process by the factor

$$
\tan ^{2} \beta_{\mathrm{CMS}} \times \frac{\mathrm{BR}\left(H \rightarrow \tau^{+} \tau^{-}\right)_{\mathrm{CMS}}+\mathrm{BR}\left(A \rightarrow \tau^{+} \tau^{-}\right)_{\mathrm{CMS}}}{\mathrm{BR}\left(H \rightarrow \tau^{+} \tau^{-}\right)_{\mathrm{SM}}}
$$

In the above, $\tan \beta_{\mathrm{CMS}}$ refers to the value of $\tan \beta$ on the discovery contour (for a given value of $M_{A}$ ) that was obtained in the analysis of the CMS Collaboration with $30 \mathrm{fb}^{-1}$ [58]. These $\tan \beta$ values as a function of $M_{A}$ correspond to the edge of the area in the $M_{A}-\tan \beta$ plane in which the signal $p p \rightarrow H / A+X, H / A \rightarrow \tau^{+} \tau^{-}$is visible (i.e. the upper bound of the LHC wedge region). The branching ratios $\mathrm{BR}\left(H \rightarrow \tau^{+} \tau^{-}\right)_{\mathrm{CMS}}$ and $\mathrm{BR}\left(A \rightarrow \tau^{+} \tau^{-}\right)_{\mathrm{CMS}}$ in the CMS analysis have been evaluated with HDECAY, incorporating therefore only the gluino-sbottom contribution to $\Delta_{b}$.

After including all $\Delta_{b}$ corrections, we evaluate the $p p \rightarrow H / A+X, H / A \rightarrow \tau^{+} \tau^{-}$process by rescaling the SM rate with the new factor,

$$
\frac{\tan ^{2} \beta}{\left(1+\Delta_{b}\right)^{2}} \times \frac{\operatorname{BR}\left(H \rightarrow \tau^{+} \tau^{-}\right)+\operatorname{BR}\left(A \rightarrow \tau^{+} \tau^{-}\right)}{\operatorname{BR}\left(H \rightarrow \tau^{+} \tau^{-}\right)_{\mathrm{SM}}},
$$

where $\Delta_{b}$ depends on $\tan \beta$. The quantities have been evaluted with FeynHiggs, allowing also decays into supersymmetric particles. The resulting shift in the discovery reach for the $p p \rightarrow H / A+X, H / A \rightarrow \tau^{+} \tau^{-}$channel can be obtained by demanding that eq. (36) and eq. (37) should give the same numerical result for a given value of $M_{A}$.

This procedure has been carried out in two benchmark scenarios for various values of $\mu$. The results are shown in Fig. 6] for the $m_{h}^{\max }$ scenario (left) and for the no-mixing scenario (right). The comparison of these results with the ones obtained by the CMS Collaboration $[58,61]$ shows that for positive values of $\mu$ the inclusion of the supersymmetric radiative corrections leads to a slight shift of the discovery region towards higher values of $\tan \beta$, i.e. to a small increase of the $\mathrm{LHC}$ wedge region. For $\mu=-200 \mathrm{GeV}$ the result remains approximately the same as the one obtained by the CMS Collaboration. Due to the smaller considered $\tan \beta$ values compared to the analysis of the Tevatron limits in Sect. 3.1.2, the corrections to the bottom Yukawa coupling from $\Delta_{b}$ are smaller, leading to a better perturbative behavior. As a consequence, also the curves for $\mu=-500,-1000 \mathrm{GeV}$ are shown in Fig. 6.

The change in the upper limit of the LHC wedge region due to the variation of $\mu$ does not exceed $\Delta \tan \beta \sim 8$. As explained above, this is a consequence of cancellations of the leading $\Delta_{b}$ effects in the Higgs production and the Higgs decay. Besides the residual $\Delta_{b}$ corrections, a further variation of the bounds is caused by the decays of the heavy Higgs bosons into supersymmetric particles. For a given value of $\mu$, the rates of these decay modes are strongly dependent on the particular values of the weak gaugino mass parameters $M_{2}$ and $M_{1}$. In our analysis, we have taken $M_{2}=200 \mathrm{GeV}$, as established by the benchmark scenarios defined in Ref. [4], while $M_{1} \simeq 100 \mathrm{GeV}$. Since the Higgs couplings to neutralinos and charginos depend strongly on the admixture between higgsino and gaugino states, the rate of these processes is strongly suppressed for large values of $|\mu| \gtrsim 500 \mathrm{GeV}$. In general, the effects of the decays $H / A \rightarrow \tilde{\chi}_{i}^{0} \tilde{\chi}_{j}^{0}, \tilde{\chi}_{k}^{ \pm} \tilde{\chi}_{l}^{\mp}$ only play a role for $M_{A} \gtrsim|\mu|+M_{1}$. Outside this range the cancellations of the $\Delta_{b}$ effects result in a very weak dependence of the rates on $\mu$. 

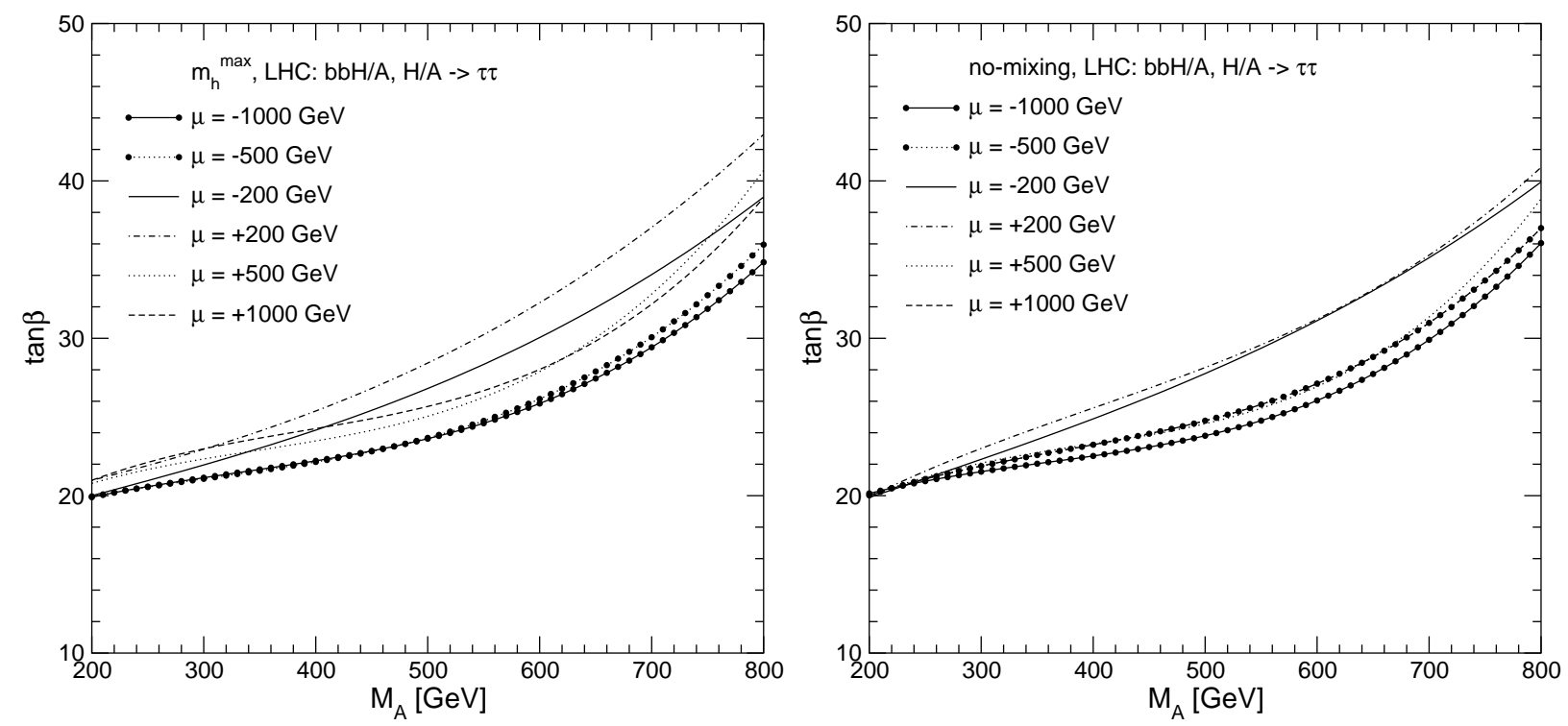

Figure 6: Variation of the $5 \sigma$ discovery potential for the $p p \rightarrow H / A+X, H / A \rightarrow \tau^{+} \tau^{-}$ process at the LHC in the $m_{h}^{\max }$ (left) and no-mixing (right) benchmark scenarios for different values of $\mu$.

The combination of the effects from supersymmetric radiative corrections and decay modes into supersymmetric particles gives rise to a rather complicated dependence of the discovery contour on $\mu$. This is illustrated in Fig. (7) (left), where the discovery contour for the $p p \rightarrow H / A+X, H / A \rightarrow \tau^{+} \tau^{-}$process is shown as a function of $\mu$ in the $m_{h}^{\max }$ scenario for different values of $M_{A}$. As explained above, for $M_{A} \gtrsim|\mu|+M_{1}$ the decay modes into supersymmetric particles have a significant impact, while outside this region the dependence on $\mu$ is rather weak.

In Fig. 8 (left) we show the results for the constrained- $m_{h}^{\max }$ scenario, see eq. (32). The variation of the discovery contour in the $M_{A}-\tan \beta$ plane with $\mu$ is completely driven in this case by the additional decay channels of the heavy Higgs bosons into charginos and neutralinos. Correspondingly, the weakest sensitivity is obtained for the smallest values of $|\mu|, \mu= \pm 200 \mathrm{GeV}$.

\subsubsection{Discovery region for the process $t H^{ \pm}, H^{ \pm} \rightarrow \tau \nu_{\tau}$}

For this process we also refer to the analysis carried out by the CMS Collaboration [58,65]. The corresponding analyses of the ATLAS Collaboration can be found in Refs. $[57,66]$. The results of the CMS Collaboration were given for an integrated luminosity of $30 \mathrm{fb}^{-1}$ in the $M_{A}-\tan \beta$ plane using the $m_{h}^{\max }$ scenario with $\mu=-200 \mathrm{GeV}$. No $\Delta_{b}$ corrections were included in the $g b \rightarrow t H^{ \pm}$production process [67] and the $H^{ \pm} \rightarrow \tau \nu_{\tau}$ decay [63].

In Fig. 9] we investigate the impact of including the $\Delta_{b}$ corrections into the production and decay processes and of varying $\mu$. In order to rescale the original result for the discovery reach in $\tan \beta$ we have first evaluated the $\tan \beta$ dependence of the production and decay processes. If no supersymmetric radiative corrections are included, for a fixed $M_{A}$ value, the 

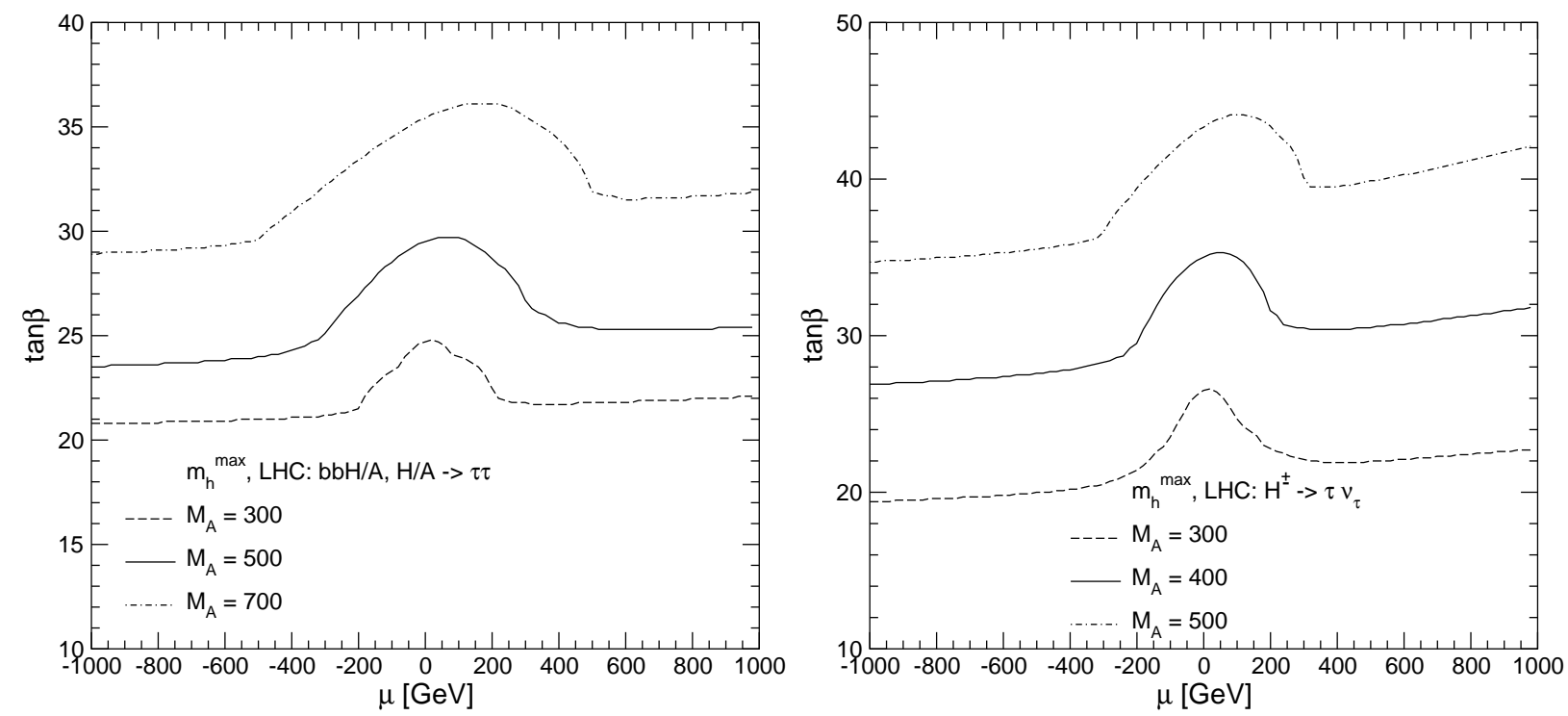

Figure 7: Variation of the $5 \sigma$ discovery contours as a function of the parameter $\mu$ in the $m_{h}^{\max }$ scenario for the $p p \rightarrow H / A+X, H / A \rightarrow \tau^{+} \tau^{-}$process (left) and the $H^{ \pm} \rightarrow \tau \nu_{\tau}$ process (right).
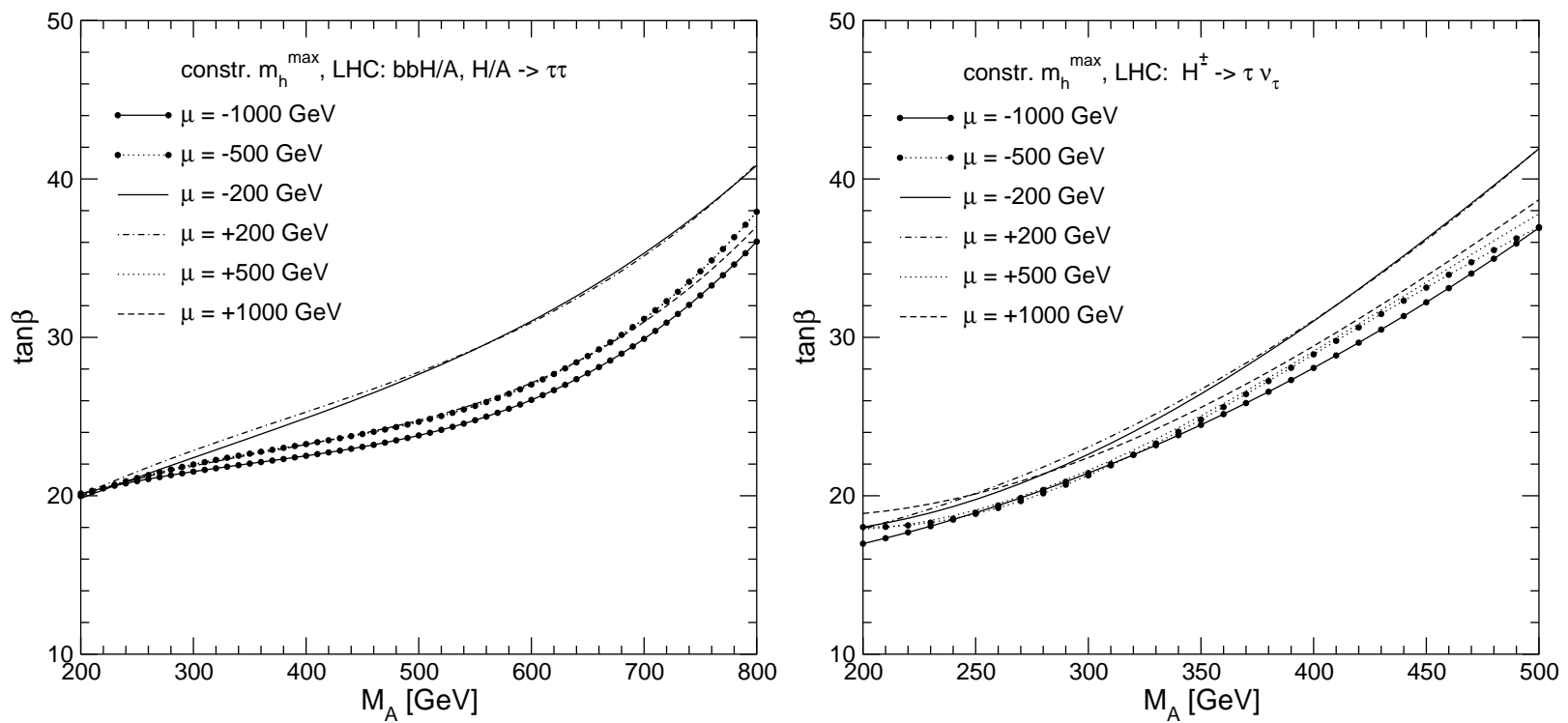

Figure 8: Variation of the $5 \sigma$ discovery contours for different values of $\mu$ in the constrained$m_{h}^{\max }$ scenario for the $p p \rightarrow H / A+X, H / A \rightarrow \tau^{+} \tau^{-}$process (left) and the $H^{ \pm} \rightarrow \tau \nu_{\tau}$ process (right). 

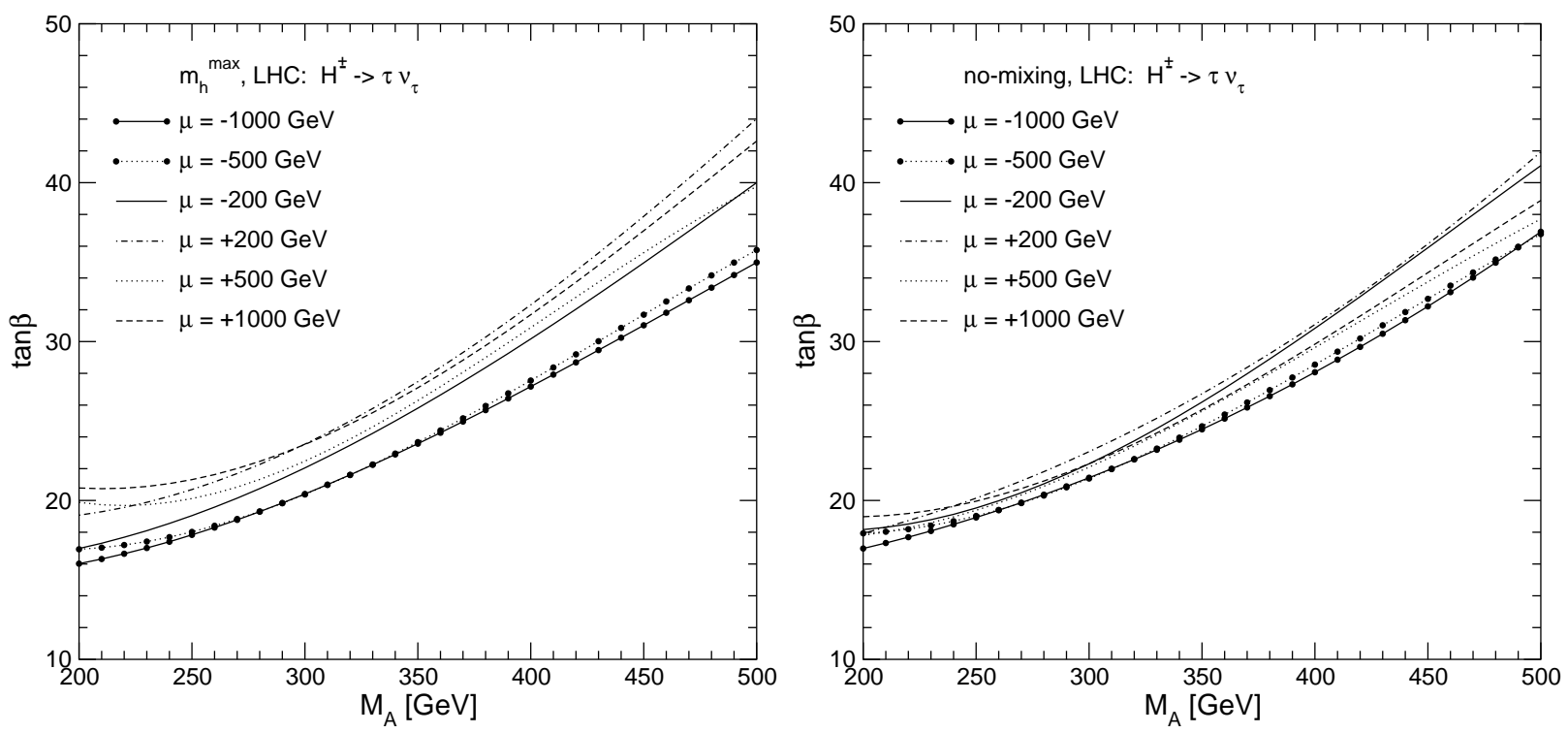

Figure 9: Variation of the $5 \sigma$ discovery contours obtained from the $t H^{ \pm}, H^{ \pm} \rightarrow \tau \nu_{\tau}$ channel in the $m_{h}^{\max }$ (left) and no-mixing (right) benchmark scenarios for different values of $\mu$.

discovery potential can be inferred by using a rate approximately proportional to

$$
\tan ^{2} \beta_{\mathrm{CMS}} \times \mathrm{BR}\left(H^{ \pm} \rightarrow \tau \nu_{\tau}\right)_{\mathrm{CMS}}
$$

Here $\tan \beta_{\mathrm{CMS}}$ is given by the edge of the area in the $M_{A}-\tan \beta$ plane in which the signal $H^{ \pm} \rightarrow \tau \nu_{\tau}$ is visible, as obtained in the CMS analysis. The $\operatorname{BR}\left(H^{ \pm} \rightarrow \tau \nu_{\tau}\right)_{\mathrm{CMS}}$ has been evaluated with HDECAY.

The rescaled result for the discovery contour, including all relevant $\Delta_{b}$ corrections, is obtained by demanding that the contribution

$$
\frac{\tan ^{2} \beta}{\left(1+\Delta_{b}\right)^{2}} \times \operatorname{BR}\left(H^{ \pm} \rightarrow \tau \nu_{\tau}\right)
$$

where $\Delta_{b}$ depends on $\tan \beta$, is numerically equal to the one of eq. (38). The quantities in eq. (39) have been evaluated with FeynHiggs.

This procedure has been carried out in two benchmark scenarios for various values of $\mu$. The results are shown in Fig. 9] for the $m_{h}^{\max }$ scenario (left) and for the no-mixing scenario (right). As a consequence of the cancellations of the leading $\Delta_{b}$ effects in the Higgs production and the Higgs decay the change in the discovery contour due to the variation of $\mu$ does not exceed $\Delta \tan \beta \sim 10(6)$ in the $m_{h}^{\max }$ (no-mixing) scenario. Also in this case there is a variation of the contour caused by decays into supersymmetric particles that, as in the neutral Higgs boson case, are only relevant for small values of $|\mu|$. For completeness, in Fig. 7 (right) we show the corresponding variation of the discovery contour for the $m_{h}^{\max }$ scenario as a function of $\mu$, for different values of $M_{A}$. Outside the region where the decays into supersymmetric particles are relevant the dependence on $\mu$ is relatively weak, but somewhat more pronounced than for the case of the neutral Higgs bosons $H$ and $A$. 
In Fig. 8 (right) we show the results for the constrained- $m_{h}^{\max }$ scenario, see eq. (32). As in the case of the neutral Higgs bosons, the variation of the discovery contour in the $M_{A}-\tan \beta$ plane is completely driven by the additional decay channels of the heavy Higgs bosons into charginos and neutralinos.

\section{Benchmark Scenarios}

The benchmark scenarios defined in Ref. [4], which were mainly designed for the search for the light $\mathcal{C P}$-even Higgs boson $h$ in the $\mathcal{C P}$-conserving case, are also useful in the search for the heavy MSSM Higgs bosons $H, A$ and $H^{ \pm}$. In order to take into account the dependence on $\mu$, which as explained above is particularly pronounced for the $b \bar{b} \phi, \phi \rightarrow b \bar{b}$ channel, we suggest to extend the definition of the $m_{h}^{\max }$ and no-mixing scenarios given in Ref. [4] by several discrete values of $\mu$. The scenarios defined in Ref. [4] read

$\underline{m_{h}^{\max }:}$

$$
\begin{aligned}
m_{t} & =174.3 \mathrm{GeV}, \\
M_{\mathrm{SUSY}} & =1000 \mathrm{GeV}, \\
\mu & =200 \mathrm{GeV}, \\
M_{2} & =200 \mathrm{GeV}, \\
X_{t}^{\mathrm{OS}} & =2 M_{\mathrm{SUSY}}(\text { FD calculation }), \\
X_{t}^{\mathrm{MS}} & =\sqrt{6} M_{\mathrm{SUSY}}(\text { RG calculation }) \\
A_{b} & =A_{t}, \\
m_{\tilde{g}} & =0.8 M_{\mathrm{SUSY}} .
\end{aligned}
$$

no-mixing:

$$
\begin{aligned}
m_{t} & =174.3 \mathrm{GeV}, \\
M_{\mathrm{SUSY}} & =2000 \mathrm{GeV}, \\
\mu & =200 \mathrm{GeV}, \\
M_{2} & =200 \mathrm{GeV}, \\
X_{t} & =0(\mathrm{FD} / \text { RG calculation }) \\
A_{b} & =A_{t}, \\
m_{\tilde{g}} & =0.8 M_{\mathrm{SUSY}}
\end{aligned}
$$

$\underline{\text { constrained } m_{h}^{\max }}$ :

$$
\begin{aligned}
m_{t} & =174.3 \mathrm{GeV}, \\
M_{\mathrm{SUSY}} & =1000 \mathrm{GeV}, \\
\mu & =200 \mathrm{GeV}, \\
M_{2} & =200 \mathrm{GeV}, \\
X_{t}^{\mathrm{OS}} & =-2 M_{\mathrm{SUSY}}(\text { FD calculation }), \\
X_{t}^{\mathrm{MS}} & =-\sqrt{6} M_{\mathrm{SUSY}} \text { (RG calculation) }, \\
A_{b} & =A_{t}, \\
m_{\tilde{g}} & =0.8 M_{\mathrm{SUSY}} .
\end{aligned}
$$


The constrained- $m_{h}^{\max }$ scenario differs from eq. (40) only by the reversed sign of $X_{t}$. While the positive sign of the product $\left(\mu M_{2}\right)$ results in general in better agreement with the $(g-2)_{\mu}$ experimental results, the negative sign of the product $\left(\mu A_{t}\right)$ yields in general (assuming minimal flavor violation) better agreement with the $\mathrm{BR}(b \rightarrow s \gamma)$ measurements.

Motivated by the analysis in Sect. 3 we suggest to investigate the following values of $\mu$

$$
\mu= \pm 200, \pm 500, \pm 1000 \mathrm{GeV}
$$

allowing both an enhancement and a suppression of the bottom Yukawa coupling and taking into account the limits from direct searches for charginos at LEP [68]. As discussed above, the results in the constrained- $m_{h}^{\max }$ scenario are expected to yield more robust bounds against the variation of $\mu$ than in the other scenarios. It should be noted that the values $\mu=$ $-500,-1000 \mathrm{GeV}$ can lead to such a large enhancement of the bottom Yukawa coupling that a perturbative treatment is no longer possible in the region of very large values of $\tan \beta$. Some care is therefore necessary to assess up to which values of $\mu$ reliable results can be obtained, see e.g. the discussion of Fig. 5 .

The value of the top-quark mass in Ref. [4] was chosen according to the experimental central value at that time. We propose to substitute this value with the most up-to-date experimental central value for $m_{t}$.

\section{Conclusions}

In this paper we have analyzed the impact of supersymmetric radiative corrections on the current MSSM Higgs boson exclusion limits at the Tevatron and the prospective discovery reach at the LHC. In particular, we have studied the variation of the exclusion and discovery contours obtained in different MSSM benchmark scenarios under changes of the higgsino mass parameter $\mu$ and the supersymmetry breaking parameters associated with the third generation squarks. These parameters determine the most important supersymmetric radiative corrections in the large $\tan \beta$ region that are associated with a change of the effective Yukawa couplings of the bottom quarks to the Higgs fields (since the squarks are relatively heavy in the considered benchmark scenarios, other squark-loop effects are sub-dominant). These corrections had been ignored or only partially considered in some of the previous analyses of Higgs searches at hadron colliders. We have shown that their inclusion leads to a significant modification of the discovery and exclusion regions.

We have investigated the exclusion bounds obtained from the Tevatron searches for non SM-like Higgs bosons in different channels. For the $b \bar{b} \phi, \phi \rightarrow b \bar{b}$ channel $(\phi=h, H, A)$ we find that the effects of the supersymmetric radiative corrections on the exclusion bounds in the $M_{A}$-tan $\beta$ plane are quite dramatic. While in the $m_{h}^{\max }$ scenario the current data allow to rule out values of $\tan \beta \gtrsim 50$ (35) for $M_{A} \approx 100 \mathrm{GeV}$ if the higgsino mass parameter is chosen as $\mu=-200 \mathrm{GeV}(-1000 \mathrm{GeV})$, hardly any bound on $\tan \beta$ can be set if positive values of $\mu$ are chosen. The shifts are smaller, but still important, for the no-mixing benchmark scenario. We have shown that the constrained $-m_{h}^{\max }$ scenario yields results that are much more stable against variations of $\mu$ than the other benchmark scenarios.

For the inclusive channel with $\tau^{+} \tau^{-}$final states, $p \bar{p} \rightarrow \phi \rightarrow \tau^{+} \tau^{-}$, compensations occur between large corrections to Higgs production and decay, so that the limits in the $M_{A}-\tan \beta$ 
plane obtained from this channel turn out to be less affected by varying $\mu$ than the ones from the associated production with bottom quarks. Nevertheless we have found that the exclusion limit is shifted by up to $\Delta \tan \beta=30$ as a consequence of choosing different input values for $\mu$. We have investigated the impact of including the dominant supersymmetric radiative corrections to the gluon fusion production process, which had previously been omitted. The inclusion of these corrections leads to a shift of up to $\Delta \tan \beta=10$ in the exclusion limit. Following our analysis, the CDF Collaboration has adopted the prescription outlined in this paper for incorporating the correction into the $g g \rightarrow \phi$ production process. The Tevatron experiments are expected to collect further data at higher luminosities, up to $4-8 \mathrm{fb}^{-1}$, in the next few years. This will extend the Tevatron MSSM Higgs boson discovery and exclusion reach in the $M_{A}$-tan $\beta$ plane to lower values of $\tan \beta$, decreasing the sensitivity of the obtained bounds to variations of the low energy supersymmetry mass parameters.

For the LHC we have analyzed the channels $p p \rightarrow H / A+X, H / A \rightarrow \tau^{+} \tau^{-}$and $t H^{ \pm}, H^{ \pm} \rightarrow \tau \nu_{\tau}$, which yield the best sensitivities in the search for heavy MSSM Higgs bosons. Accordingly, the discovery contours for these channels in the $M_{A}$-tan $\beta$ plane determine the boundary of the region where only the (SM-like) light $\mathcal{C} \mathcal{P}$-even Higgs boson can be detected at the LHC. Since the discovery contours for the LHC are at smaller values of $\tan \beta$ compared to those accessible via the current exclusion bounds at the Tevatron, the impact of the $\tan \beta$-enhanced supersymmetric corrections is less pronounced in this case. We have studied the effect of including the dominant supersymmetric corrections, which had been omitted in the analyses of the production processes at the LHC, and their variation with the relevant parameters. Possible decays of the heavy MSSM Higgs bosons into charginos and neutralinos have been taken into account. We have found that the prospective discovery contours at the LHC are shifted by up to $\Delta \tan \beta \lesssim 10$.

Based on our analysis of the sensitivities of the searches for MSSM Higgs bosons at the Tevatron and the LHC we have defined benchmark scenarios for the analysis of MSSM Higgsboson searches at hadron colliders. They are based on a generalization of similar benchmark scenarios proposed for the searches for SM-like MSSM Higgs bosons at the Tevatron and the LHC.

\section{Acknowledgements}

M. C., C.E.M. W. and G. W. thank the Aspen Center for Physics for its hospitality, where part of this work was done. We thank A. Anastassov, J. Conway, A. Goussiou, A. Haas, B. Heinemann, A. Kharchilava, R. Kinnunen, A. Lath, A. Nikitenko, T. Plehn, M. Schumacher and M. Spira for helpful discussions. 


\section{References}

[1] [LEP Higgs working group], Phys. Lett. B 565 (2003) 61, hep-ex/0306033.

[2] [LEP Higgs working group], hep-ex/0107030; hep-ex/0107031; LHWG Note 2004-1, see: lephiggs . web . cern . ch/LEPHIGGS/papers .

[3] M. Carena, S. Heinemeyer, C. Wagner and G. Weiglein, hep-ph/9912223.

[4] M. Carena, S. Heinemeyer, C. Wagner and G. Weiglein, Eur. Phys. J. C 26 (2003) 601, hep-ph/0202167.

[5] S. Heinemeyer, W. Hollik and G. Weiglein, JHEP 0006 (2000) 009, hep-ph/9909540.

[6] M. Schumacher, Czech. J. Phys. 54 (2004) A103.

[7] V. Abazov et al. [D0 Collaboration], hep-ex/0504018.

[8] A. Abulencia et al. [CDF Collaboration], hep-ex/0508051.

[9] [CDF Collaboration], hep-ex/0510065,

R. Eusebi, Ph.d. thesis: "Search for charged Higgs in $t \bar{t}$ decay products from protonantiproton collisions at $\sqrt{s}=1.96 \mathrm{TeV}$ ", University of Rochester, 2005 .

[10] A. Belyaev, J. Pumplin, W. Tung and C. Yuan, hep-ph/0508222.

[11] M. Carena, P. Chankowski, S. Pokorski and C. Wagner, Phys. Lett. B 441 (1998) 205, hep-ph/9805349.

[12] S. Heinemeyer, W. Hollik and G. Weiglein, Eur. Phys. J. C 9 (1999) 343, hep-ph/9812472.

[13] J. Ellis, G. Ridolfi and F. Zwirner, Phys. Lett. B 257 (1991) 83;

Y. Okada, M. Yamaguchi and T. Yanagida, Prog. Theor. Phys. 85 (1991) 1;

H. Haber and R. Hempfling, Phys. Rev. Lett. 66 (1991) 1815.

[14] A. Brignole, Phys. Lett. B 281 (1992) 284.

[15] P. Chankowski, S. Pokorski and J. Rosiek, Phys. Lett. B 286 (1992) 307; Nucl. Phys. B 423 (1994) 437, hep-ph/9303309.

[16] A. Dabelstein, Nucl. Phys. B 456 (1995) 25, hep-ph/9503443, Z. Phys. C 67 (1995) 495, hep-ph/9409375.

[17] R. Hempfling and A. Hoang, Phys. Lett. B 331 (1994) 99, hep-ph/9401219.

[18] J. Casas, J. Espinosa, M. Quirós and A. Riotto, Nucl. Phys. B 436 (1995) 3, E: ibid. B 439 (1995) 466, hep-ph/9407389.

[19] M. Carena, J. Espinosa, M. Quirós and C. Wagner, Phys. Lett. B 355 (1995) 209, hep-ph/9504316.

M. Carena, M. Quirós and C. Wagner, Nucl. Phys. B 461 (1996) 407, hep-ph/9508343 
[20] H. Haber, R. Hempfling and A. Hoang, Z. Phys. C 75 (1997) 539, hep-ph/9609331.

[21] S. Heinemeyer, W. Hollik and G. Weiglein, Phys. Rev. D 58 (1998) 091701, hep-ph/9803277, Phys. Lett. B 440 (1998) 296, hep-ph/9807423.

[22] R. Zhang, Phys. Lett. B 447 (1999) 89, hep-ph/9808299;

J. Espinosa and R. Zhang, JHEP 0003 (2000) 026, hep-ph/9912236.

[23] G. Degrassi, P. Slavich and F. Zwirner, Nucl. Phys. B 611 (2001) 403, hep-ph/0105096

[24] J. Espinosa and R. Zhang, Nucl. Phys. B 586 (2000) 3, hep-ph/0003246.

[25] A. Brignole, G. Degrassi, P. Slavich and F. Zwirner, Nucl. Phys. B 631 (2002) 195, hep-ph/0112177.

[26] A. Brignole, G. Degrassi, P. Slavich and F. Zwirner, Nucl. Phys. B 643 (2002) 79, hep-ph/0206101.

[27] S. Heinemeyer, W. Hollik, H. Rzehak and G. Weiglein, Eur. Phys. J. C 39 (2005) 465, hep-ph/0411114, hep-ph/0506254.

[28] R. Hempfling, Phys. Rev. D 49 (1994) 6168;

L. Hall, R. Rattazzi and U. Sarid, Phys. Rev. D 50 (1994) 7048, hep-ph/9306309,

M. Carena, M. Olechowski, S. Pokorski and C. Wagner, Nucl. Phys. B 426 (1994) 269, hep-ph/9402253.

[29] M. Carena, D. Garcia, U. Nierste and C. Wagner, Nucl. Phys. B 577 (2000) 577, hep-ph/9912516.

[30] H. Eberl, K. Hidaka, S. Kraml, W. Majerotto and Y. Yamada, Phys. Rev. D 62 (2000) 055006, hep-ph/9912463.

[31] G. Degrassi, A. Dedes and P. Slavich, Nucl. Phys. B 672 (2003) 144, hep-ph/0305127.

[32] G. Degrassi, S. Heinemeyer, W. Hollik, P. Slavich and G. Weiglein, Eur. Phys. J. C 28 (2003) 133, hep-ph/0212020.

[33] S. Heinemeyer, W. Hollik and G. Weiglein, hep-ph/0412214.

[34] S. Heinemeyer, W. Hollik and G. Weiglein, Comput. Phys. Comm. 124 (2000) 76, hep-ph/9812320 hep-ph/0002213; see: www.feynhiggs.de .

[35] M. Frank, S. Heinemeyer, W. Hollik and G. Weiglein, hep-ph/0202166.

[36] T. Hahn, S. Heinemeyer, W. Hollik and G. Weiglein, hep-ph/0507009.

[37] M. Frank, T. Hahn, S. Heinemeyer, W. Hollik, H. Rzehak and G. Weiglein, in preparation.

[38] J. Lee, A. Pilaftsis et al., Comput. Phys. Comm. 156 (2004) 283, hep-ph/0307377. 
[39] M. Carena, H. Haber, S. Heinemeyer, W. Hollik, C. Wagner, and G. Weiglein, Nucl. Phys. B 580 (2000) 29, hep-ph/0001002.

[40] A. Pilaftsis, Phys. Rev. D 58 (1998) 096010, hep-ph/9803297;

A. Pilaftsis and C. Wagner, Nucl. Phys. B 553 (1999) 3, hep-ph/9902371;

M. Carena, J. Ellis, A. Pilaftsis and C. Wagner, Nucl. Phys. B 586 (2000) 92, hep-ph/0003180, Nucl. Phys. B 625 (2002) 345, hep-ph/0111245.

M. Carena, J. Ellis, S. Mrenna, A. Pilaftsis and C. Wagner, Nucl. Phys. B 659 (2003) 145, hep-ph/0211467;

S. Choi, M. Drees and J. Lee, Phys. Lett. B 481 (2000) 57, hep-ph/0002287.

[41] S. Heinemeyer, Eur. Phys. J. C 22 (2001) 521, hep-ph/0108059;

M. Frank, S. Heinemeyer, W. Hollik and G. Weiglein, hep-ph/0212037, appeared in the proceedings of SUSY02, DESY, Hamburg, Germany, July 2002.

[42] S. Heinemeyer, hep-ph/0407244.

[43] S. Martin, Phys. Rev. D 65 (2002) 116003, hep-ph/0111209, Phys. Rev. D 66 (2002) 096001, hep-ph/0206136 Phys. Rev. D 67 (2003) 095012, hep-ph/0211366 Phys. Rev. D 68 (2003) 075002, hep-ph/0307101; Phys. Rev. D 70 (2004) 016005, hep-ph/0312092; Phys. Rev. D 71 (2005) 016012, hep-ph/0405022 Phys. Rev. D 71 (2005) 116004, hep-ph/0502168;

S. Martin and D. Robertson, hep-ph/0501132

[44] S. Heinemeyer, W. Hollik and G. Weiglein, Phys. Lett. B 455 (1999) 179, hep-ph/9903404

[45] J. Guasch, P. Häfliger and M. Spira, Phys. Rev. D 68 (2003) 115001, hep-ph/0305101.

[46] S. Dawson and S. Willenbrock, Phys. Rev. D 40 (1989) 2880;

S. Dawson, Nucl. Phys. B 359 (1991) 283;

A. Djouadi, M. Spira and P. Zerwas, Phys. Lett. B 264 (1991) 440;

S. Catani, D. de Florian and M. Grazzini, JHEP 0105 (2001) 025, hep-ph/0102227;

R. Harlander and W. Kilgore, Phys. Rev. Lett. 88 (2002) 201801, hep-ph/0201206;

C. Anastasiou and K. Melnikov, Nucl. Phys. B 646 (2002) 220, hep-ph/0207004

V. Ravindran, J. Smith and W. van Neerven, Nucl. Phys. B 665 (2003) 325, hep-ph/0302135.

[47] R. Harlander and M. Steinhauser, Phys. Lett. B 574 (2003) 258, hep-ph/0307346 JHEP 0409 (2004) 066, hep-ph/0409010.

[48] J. Campbell, R. Ellis, F. Maltoni and S. Willenbrock, Phys. Rev. D 67 (2003) 095002, hep-ph/0204093.

[49] S. Dittmaier, M. Krämer and M. Spira, Phys. Rev. D 70 (2004) 074010, hep-ph/0309204.

S. Dawson, C. Jackson, L. Reina and D. Wackeroth, Phys. Rev. D 69 (2004) 074027, hep-ph/0311067; Phys. Rev. Lett. 94 (2005) 031802, hep-ph/0408077;

J. Campbell et al., hep-ph/0405302. 
[50] R. Harlander and W. Kilgore, Phys. Rev. D 68 (2003) 013001, hep-ph/0304035.

[51] S. Gorishny, A. Kataev, S. Larin and L. Surguladze, Mod. Phys. Lett. A 5 (1990) 2703; Phys. Rev. D 43 (1991) 1633;

A. Kataev and V. Kim, Mod. Phys. Lett. A 9 (1994) 1309;

L. Surguladze, Phys. Lett. B 338 (1994) 229, hep-ph/9406294, Phys. Lett. B 341 (1994) 60, hep-ph/9405325

K. Chetyrkin, Phys. Lett. B 390 (1997) 309, hep-ph/9608318

K. Chetyrkin and A. Kwiatkowski, Nucl. Phys. B 461 (1996) 3, hep-ph/9505358

S. Larin, T. van Ritbergen and J. Vermaseren, Phys. Lett. B 362 (1995) 134, hep-ph/9506465,

P. Chankowski, S. Pokorski and J. Rosiek, Nucl. Phys. B 423 (1994) 497;

S. Heinemeyer, W. Hollik and G. Weiglein, Eur. Phys. J. C 16 (2000) 139, hep-ph/0003022.

[52] M. Carena, S. Mrenna and C. Wagner, Phys. Rev. D 60 (1999) 075010, hep-ph/9808312, Phys. Rev. D 62 (2000) 055008, hep-ph/9907422.

[53] A. Belyaev, D. Garcia, J. Guasch and J. Sola, JHEP 0206 (2002) 059, hep-ph/0203031.

[54] A. Belyaev, A. Blum, R. Chivukula and E. Simmons, hep-ph/0506086

[55] M. Spira, hep-ph/9510347.

[56] [CDF Collaboration], CDF note 7161.

[57] ATLAS Collaboration, Detector and Physics Performance Technical Design Report, CERN/LHCC/99-15 (1999), see: atlasinfo.cern.ch/Atlas/GROUPS/PHYSICS/TDR/access.html .

[58] S. Abdullin et al., Eur. Phys. J. C 39S2 (2005) 41.

[59] A. Datta, A. Djouadi, M. Guchait and F. Moortgat, Nucl. Phys. B 681 (2004) 31, hep-ph/0303095.

[60] F. Moortgat in G. Weiglein et al. [LHC / LC Study Group], hep-ph/0410364.

[61] R. Kinnunen and A. Nikitenko, CMS note 2003/006.

[62] J. Thomas, ATL-PHYS-2003-003;

D. Cavalli and D. Negri, ATL-PHYS-2003-009.

[63] A. Djouadi, J. Kalinowski and M. Spira, Comput. Phys. Comm. 108 (1998) 56, hep-ph/9704448.

[64] See: people.web.psi.ch/spira/hqq .

[65] R. Kinnunen, P. Salmi and N. Stepanov, CMS note 2002/024. 
[66] K. Assamagan, Y. Coadou and A. Deandrea, Eur. Phys. J. directC 4 (2002) 9, hep-ph/0203121;

K. Assamagan and N. Gollub, Eur. Phys. J. C 39S2 (2005) 25, hep-ph/0406013.

[67] R. Kinnunen, S. Nikitenko and T. Plehn, private communication.

[68] G. Abbiendi et al. [OPAL Collaboration], Eur. Phys. J. C 35 (2004) 1, hep-ex/0401026. 